\title{
Synapses of active galactic nuclei:
}

\section{Comparing X-ray and optical classifications using artificial neural networks ${ }^{\star}$}

\author{
O. González-Martín ${ }^{1,2, \star \star}$, D. Díaz-González ${ }^{3}$, J. A. Acosta-Pulido ${ }^{1,2}$, J. Masegosa $^{4}$, \\ I. E. Papadakis ${ }^{5,6}$, J. M. Rodríguez-Espinosa ${ }^{1,2}$, I. Márquez ${ }^{4}$, and L. Hernández-García ${ }^{4}$ \\ ${ }^{1}$ Instituto de Astrofísica de Canarias (IAC), C/Vía Láctea s/n, 38205 La Laguna, Spain \\ e-mail: omairagm@iac.es \\ 2 Departamento de Astrofísica, Universidad de La Laguna (ULL), 38205 La Laguna, Spain \\ 3 Shidix Technologies, 38320, La Laguna, Spain \\ ${ }^{4}$ Instituto de Astrofísica de Andalucía, CSIC, C/ Glorieta de la Astronomía s/n, 18005 Granada, Spain \\ 5 Physics Department, University of Crete, PO Box 2208, 71003 Heraklion, Crete, Greece \\ ${ }^{6}$ IESL, Foundation for Research and Technology, 71110 Heraklion, Crete, Greece
}

Received 2 September 2013 / Accepted 3 April 2014

\section{ABSTRACT}

\begin{abstract}
Context. Many classes of active galactic nuclei (AGN) have been defined entirely through optical wavelengths, while the X-ray spectra have been very useful to investigate their inner regions. However, optical and X-ray results show many discrepancies that have not been fully understood yet.

Aims. The main purpose of the present paper is to study the synapses (i.e., connections) between X-ray and optical AGN classifications.

Methods. For the first time, the newly implemented EFLUXER task allowed us to analyse broad band X-ray spectra of a sample of emission-line nuclei without any prior spectral fitting. Our sample comprises 162 spectra observed with XMM-Newton/pn of 90 local emission line nuclei in the Palomar sample. It includes, from the optical point of view, starbursts (SB), transition objects (T2), low-ionisation nuclear emission line regions (L1.8 and L2), and Seyfert nuclei (S1, S1.8, and S2). We used artificial neural networks (ANNs) to study the connection between X-ray spectra and optical classes.

Results. Among the training classes, the ANNs are $90 \%$ efficient at classifying the S1, S1.8, and SB classes. The S1 and S1.8 classes show a negligible SB-like component contribution with a wide range of contributions from S1- and S1.8-like components. We suggest that this broad range of values is related to the high degree of obscuration in the X-ray regime. When including all the objects in our sample, the S1, S1.8, S2, L1.8, L2/T2/SB-AGN (SB with indications of AGN activity in the literature), and SB classes have similar average X-ray spectra, but these average spectra can be distinguished from class to class. The S2 (L1.8) class is linked to the S1.8 (S1) class with a larger SB-like component than the S1.8 (S1) class. The L2, T2, and SB-AGN classes constitute a class in the X-rays similar to the S2 class, albeit with larger portions of SB-like component. We argue that this SB-like component might come from the contribution of the host galaxy emission to the X-rays, which is high when the AGN is weak. Up to $80 \%$ of the emission line nuclei and, on average, all the optical classes included in our sample show a significant fraction of S1-like or S1.8-like components. Thus, an AGN-like component seems to be present in the vast majority of the emission line nuclei in our sample.

Conclusions. The ANN trained in this paper is not only useful for studying the synergies between the optical and X-ray classifications, but might also be used to infer optical properties from X-ray spectra in surveys like eRosita.
\end{abstract}

Key words. galaxies: active - galaxies: Seyfert $-\mathrm{X}$-rays: galaxies

\section{Introduction}

At optical wavelengths, emission line galaxies can be grouped into HII nuclei, active galactic nuclei (AGN), galaxies with low-ionisation nuclear emission line regions (LINERs), and transition objects (whose optical spectra are intermediate between those of pure LINERs and HII regions; see Ho 2008, for a review). Optical spectroscopic studies have shown that only $10 \%$ of nearby galaxies are Seyferts, while there are no more than $20 \%$ of LINERs and $10 \%$ of transition objects (e.g., Palomar survey by Ho et al. 1997).

\footnotetext{
* Table 1 and Appendices are available in electronic form at http://www . aanda.org

$\star \star$ Juan de la Cierva fellow.
}

HII nuclei are powered by a compact star-forming region. In AGN, the main energy source is assumed to be accretion of matter into a super-massive black hole (SMBH). The nature of the main energy source in LINERs (and transition objects) is not clearly understood yet. They might be low-luminosity AGN (LLAGN), in which case, they will constitute the main fraction of the AGN population (Heckman 1980; Ho et al. 1997). However, other emission mechanisms such as shock heating (Dopita \& Sutherland 1995), OB stars in compact nuclear star clusters (Terlevich \& Melnick 1985), or pre-main-sequence star ionisation (Cid Fernandes et al. 2004) have also been proposed.

Active galactic nuclei are traditionally divided into two main classes, type 1 and type 2 objects, based on the whether (type 1) or not (type 2) there are broad permitted lines ( $F W H M>$ $2000 \mathrm{~km} \mathrm{~s}^{-1}$ ). The so-called unification model (UM) proposes that the two types of AGN are essentially the same objects 
viewed at different angles (Antonucci 1993; Urry \& Padovani 1995). An optically thick dusty torus surrounding the central source would be responsible for blocking the region where these broad emission lines are produced (the broad-line region, BLR) in type 2 Seyferts. Therefore, type 2 Seyferts are essentially type 1 Seyferts blocked by the dusty torus along the line of sight (LOS) to the observer. A strong observational evidence in favour of a unification between type 1 and type 2 Seyferts was the discovery of broad optical lines in the polarised spectrum of the archetypal type 2 Seyfert, NGC 1068 (Antonucci \& Miller 1985). The torus must not be spherically symmetric to obscure the BLR, so that at the same time the region producing the permitted narrow lines (known as narrow-line region, NLR) reaches us from the same LOS. The locus of this obscuring material was initially postulated at parsec scales and confirmed by modelling the spectral energy distribution (SED) of Seyferts (e.g., Ramos Almeida et al. 2011; Alonso-Herrero et al. 2011) and by interferometric observations (e.g., Circinus galaxy, Tristram et al. 2007). Such scales are beyond the current instrumentation, therefore the torus morphology can only be inferred by indirect measurements.

Although the UM is widely accepted for many classes of Seyferts, there is still no consensus on its general applicability for all members of each class (see Bianchi et al. 2012, for a review). An example of this mismatch is the so-called optically elusive AGN (Maiolino et al. 1998). These elusive AGN are nuclear hard X-ray sources whose intrinsic luminosities are in the Seyfert range, but lack optical Seyfert-like signatures. Another example is that about half of the brightest type 2 Seyferts are characterised by the lack of BLR even with high-quality spectropolarimetric data (known as true type 2 Seyferts, Tran 2001, 2003). These type 2 Seyferts without BLR are expected to occur at low accretion rates or low luminosities (Elitzur \& Ho 2009).

Even if LINERs are powered predominately by accretion into an SMBH, it is unclear whether the UM can also apply to these LLAGN. Indeed, both a different accretion mode and large amounts of obscuration have been proposed to explain the differences between LINERs and Seyferts (González-Martín et al. 2006, 2009a,b; Younes et al. 2011; Hernández-García et al. 2013).

X-rays in AGN are thought to originate in the innermost region of the accretion flow and are also thought to be affected by the obscuring material along the LOS. X-ray observations of AGN have provided additional evidence in favour of the UM. For example, there is substantially mode obscuring material along the LOS (measured at X-rays by the hydrogen column density, $N_{\mathrm{H}}$ ) in type 2 Seyferts than in type 1 Seyferts (e.g., Maiolino et al. 1998; Risaliti et al. 1999; Panessa et al. 2006; Cappi et al. 2006). Although modelling of X-ray spectra is one of the best ways to estimate the obscuration, this also has some caveats. For example, the obscuration measured in Seyferts depends on the model used for the underlying X-ray continuum.

The main aim of this paper is to investigate whether objects in different (optical) classes have similar X-ray spectra, and if they do, whether their average X-ray spectrum differs between the different classes or not. Furthermore, we compare the average X-ray spectra of these classes in a model independent way. Consequently, instead of fitting each individual spectrum with a suitable model, we chose to use artificial neural networks (ANNs).

We have selected for our analysis the X-ray spectra of 90 well-classified emission line nuclei included in the optically classified sample of nearby galaxies presented by Ho et al. (1997). We used ANNs to classify their X-ray spectrum and compare the average spectra of each class, without any model pre-assumptions. The main questions we address in this paper are the following: (1) how do optical classes "behave" at X-rays? In other words, do objects of the same (optical) class have the same X-ray spectrum (on average), and if so, are the average X-ray spectra of the various optical classes the same? (2) If they are different, can we understand the main physical parameter that drives those differences? Finally, (3) are AGN-like nuclei present in all emission line nuclei in nearby galaxies? Does this include galaxies that have absent or weak AGN signatures at optical wavelengths?

Section 2 gives the details on the selected sample and Sect. 3 the technical details of the reduction process. In Sect. 4 we describe the methodology, and the main results of the ANN are presented in Sect. 5. These results are discussed in Sect. 6 and summarised in Sect. 7. We assume a value of $H_{0}=75 \mathrm{~km} \mathrm{~s}^{-1} \mathrm{Mpc}^{-1}$ throughout.

\section{Sample}

We used the Palomar sample, a catalog of optical nuclear spectra reported by Ho et al. (1997). This is the largest sample of galaxy nuclei with optical spectra homogeneously observed in the nearby Universe up to date. They presented measurements of the spectroscopic parameters for 418 emission-line nuclei. The sample contains most of the bright galaxies $\left(M_{\mathrm{B}}<12\right)$ in the nearby Universe. Since our work was based on the optical classification of AGN, we considered the homogeneous analysis performed by Ho et al. (1997) as ideal for our purpose.

We obtained all the available (up to December 2012) $X_{\text {XM-Newton }}{ }^{1}$ data for the objects in the Palomar sample. We initially included 436 observations in our sample. We excluded observations where the source of interest for our analysis was out of the field of view, not detected, or close to the gap between chips in the EPIC-pn detector. We then excluded the observations for which the pile-up ${ }^{2}$ was higher than 5\% (NGC 1275, ObsID 0305780101 and NGC 4486, ObsID 0200920101). We only considered spectra with more than $~ 500$ net counts in the $0.5-10 \mathrm{keV}$ band. We imposed this restriction to include only high signal-to-noise ratio $(\mathrm{S} / \mathrm{N})$ data.

Our final sample contains 162 observations for 90 emission line nuclei. This represents $\sim 20 \%$ of the sample published by Ho et al. (1997). Table 1 shows the observational details of the X-ray data of the sample: object name (Col. 2), identifier of the observation - ObsID (Col .3), optical class (Col. 4), net exposure time (Col. 5), and net number counts (Col. 6). The optical classification is that reported in Ho et al. (1997).

Our sample includes ten S1 objects (optically classified as "S1", "S1.2", and "S1.5"), eight S1.8 objects (optically classified as "S1.8", and "S1.9"), nine S2 sources (optically classified as "S2", "S2:", and "S2::"), 11 L1.8 objects (optically classified as "L1.9"), 17 L2 objects (optically classified as "L2", "L2:", "L2::", and "S2/L"), 11 T2 objects (optically classified as "T2", "T2:", and "T2/S"), and 24 SB objects (optically classified as "H" and "H:"). The optical classes were classified by Ho et al. (1997) using BPT diagrams (named after Baldwin, Phillips $\&$ Telervich, Baldwin et al. 1981). These diagrams are based on nebular emission line ratios used to distinguish the ionisation mechanism of the ionising gas. The better-known version consists of a combination of three diagrams: [NII] $\lambda 6584 / \mathrm{H} \alpha$ versus

\footnotetext{
We used the HEASARC archive to download the data at http:// heasarc.nasa.gov

2 Pile-up occurs on X-ray CCDs when several photons hit the detector at the same place between two read-outs (Ballet 1999).
} 
[OIII] $\lambda 5007 / \mathrm{H} \beta, \quad[\mathrm{SII}] \lambda 6717,6731 / \mathrm{H} \alpha$ versus $[\mathrm{OIII}] \lambda 5007 / \mathrm{H} \beta$ and $[\mathrm{OI}] \lambda 6300 / \mathrm{H} \alpha$ versus $[\mathrm{OIII}] \lambda 5007 / \mathrm{H} \beta$. The classifications into type $1,1.2,1.5,1.8$, and 1.9 were made based on the presence and strength of broad components for $\mathrm{H} \alpha$ and $\mathrm{H} \beta$ lines. Note that here the L1.8 class refer, for consistency with the S1.8 class, to objects belonging to the L1.8 and L1.9 optical type; however, the L1.8 sample consists only of objects optically classified as L1.9.

Active galactic nuclei signatures (mostly from X-ray spectral studies) were discovered in half of the SB objects in our sample (12 out of 24), although they were classified as SB according to the classification given by Ho et al. (1997). Six S2 nuclei belong to the category of True type 2 Seyferts. Furthermore, eight objects (classified as S1, S1.8, S2, L1.8, L2, or SB) show a hydrogen column density in the Compton-thick regime (i.e. $N_{\mathrm{H}}>1.5 \times 10^{24} \mathrm{~cm}^{-2}$ ). This information, together with the corresponding references, is included in Col. 11 of Table 1.

\section{X-ray data processing}

The XMM-Newton data were reduced with the latest SAS version (v12.0.1), using the most up-to-date calibration files available. We only analysed EPIC/pn (Strüder et al. 2001) data because of the higher count rate and lower distortion due to pile-up.

Time intervals of quiescent particle-background were screened from the net source spectrum by excluding time intervals above $3 \sigma$ of the median value for the background light curve. The nuclear positions were retrieved from NED, and source counts in each case were accumulated from a circular region of radii between $15^{\prime \prime}-50^{\prime \prime}$ (300-1000 pixels). These radii were chosen to avoid nearby sources and to sample most of the PSF according to the observing mode. The background region was selected using a source-free circular region on the same CCD chip as the source with an automatic routine created with IDL. We selected only single and double pixel events (i.e., patterns of 0-4). Bad pixels and events too close to the edges of the $C C D$ chip were rejected using FLAG $=0$. The regions were extracted with the SAS EVSELECT task. pn redistribution matrix and effective areas were calculated with RMFGEN and ARFGEN tasks, respectively.

Pile-up affects both flux measurements and spectral characterisation of bright sources (Ballet 2001). The pile-up was estimated with the PIMMS software using the $0.5-10 \mathrm{keV}$ flux interval and assuming a power-law model with slope $\Gamma=2.1$ (canonical value for AGN) and the setting of each observation. Note that observations with pile-up fractions higher than $5 \%$ were previously excluded from our sample (see Sect. 2). Only two observations showed a pile-up fraction below 5\%: NGC 1275 (ObsID 0085110101) and NGC 4486 (ObsID 0114120101 ) with $3.2 \%$ and $2.2 \%$ pile-up, respectively. This means that pile-up is negligible in our sample.

The spectra were flux-calibrated using the EFLUXER task within the SAS. The final spectral range is $0.5-10.0 \mathrm{keV}$ with energy bins of $\Delta E=0.05 \mathrm{keV}$. Note that we excluded data below $0.5 \mathrm{keV}$ since EFLUXER seems to be less accurate at such energies. These final spectra are expressed in luminosity units (erg/s) and redshifted to rest-frame according to the distance of the source (see Table 1). The flux-calibrated spectra for the entire sample are provided in Appendix B.

\section{Artificial neural network}

As we explained in Sect. 1, we did not follow the standard procedure of fitting the X-ray spectra with a model to avoid the possibility that the results might be affected by model-dependent degeneracies. Instead, we chose to use ANNs. Briefly, ANNs are computing algorithms that to some extent resemble the behaviour of the brain. They consist of processing units, neurons, with multiple signal transmitter connections organised as a network. These connections have adaptable strengths, synaptic weights, which modify the signal transmitted to (and from) each neuron. The training of the network is the process of adjusting weights, so that the network learns how to solve a specific problem. We describe this process in the following subsections.

The code used to implement the ANN is the Python-based reinforcement learning, artificial intelligence and neural (PyBrain) network library (Schaul et al. 2010). PyBrain ${ }^{3}$ is a modular machine learning library for Python.

\subsection{Inputs, outputs, and the network training}

The primary inputs for this study are the X-ray spectra of the objects in our sample. These spectra were extracted using standard $\mathrm{X}$-ray procedures as explained in Sect. 3 and were then converted into physical units with the algorithm EFLUXER within the SAS.

The training process is set to classify the X-ray spectra of the sources within the SB and S1 optical classes. We chose these classes for training the network because the objects belonging to them are assumed to be representative of objects where accretion (Seyferts) and star-forming-related processes (SBs) are the main source of power, respectively. To study the connection in $\mathrm{X}$-rays between optical classes of types, one should ideally use S1 and S2 samples. However, the S2 class consists in several types of objects whose nature might be controversial. Some objects are heavily obscured (with negligible emission in X-rays), while others may lack the BLR (see Table 1 in this paper and Bianchi et al. 2012, for a review). We therefore chose to use the S1.8 sample as a third training set since it represents a more homogeneous class in X-rays than the S2 class. We therefore used the following three classes for our training sets:

- S1-training: we ascribed all the objects within the S1 class to this class. This training set includes nine AGN. We excluded NGC 1275 because of the strong contribution of the diffuse emission from the centre of the galaxy cluster (see Sanders et al. 2005).

- S1.8-training: we ascribed all the objects in the S1.8 class to this class. This training set contains seven AGN. We excluded NGC 1068 because it is a Compton-thick source and, therefore, the primary AGN emission is not seen at the energy range analysed in this study.

- SB-training: only the SB class (objects marked H in Table 1) is included in this training set, avoiding the objects classified as SB-AGN (see Sect. 2). We excluded IC 10 because this galaxy hosts a ULX included in the PSF of XMM-Newton. This training set contains 11 SBs.

For objects with more than one observation, we chose that with the highest luminosity. We tested that the selection of another observation of the same object does not substantially change the final classification. Thus, the training set contains a total of 27 spectra (one per object). All the observations used for the training process are marked TR in Col. 7 of Table 1.

The optical classification is recorded in the network outputs using a vector of three elements $v \equiv\left[v_{\mathrm{S} 1}, v_{\mathrm{S} 1.8}, v_{\mathrm{SB}}\right]$. During the training process, we used the vectors $v=[100,0,0]$,

http://pybrain.org/pages/home 
Table 2. Mean and median values for the ANN components per optical class.

\begin{tabular}{|c|c|c|c|c|c|c|}
\hline & \multicolumn{2}{|c|}{$v_{\mathrm{S} 1}$} & \multicolumn{2}{|c|}{$v_{\mathrm{S} 1.8}$} & \multicolumn{2}{|c|}{$v_{\mathrm{SB}}$} \\
\hline & Mean & Median & Mean & Median & Mean & Median \\
\hline S1 & $89.7 \pm 3.0$ & 98.0 & $12.1 \pm 2.9$ & 3.3 & $-2.3 \pm 2.4$ & -3.1 \\
\hline S1.8 & $24.2 \pm 7.7$ & 10.5 & $65.8 \pm 9.0$ & 78.7 & $9.0 \pm 2.6$ & 6.4 \\
\hline $\mathrm{S} 2$ & $10.8 \pm 7.4$ & 3.9 & $33.0 \pm 7.3$ & 36.8 & $51.1 \pm 8.5$ & 49.9 \\
\hline L1.8 & $29.6 \pm 5.2$ & 33.2 & $21.4 \pm 6.2$ & 10.5 & $47.6 \pm 7.4$ & 49.0 \\
\hline L2 & $1.0 \pm 3.9$ & 4.3 & $16.1 \pm 2.7$ & 15.2 & $78.1 \pm 3.6$ & 79.5 \\
\hline $\mathrm{T} 2$ & $5.0 \pm 5.5$ & 8.7 & $18.1 \pm 2.7$ & 19.4 & $72.4 \pm 6.3$ & 66.5 \\
\hline SB-AGN & $7.1 \pm 2.8$ & 9.7 & $16.9 \pm 3.2$ & 17.0 & $74.2 \pm 2.5$ & 75.1 \\
\hline SB & $8.7 \pm 2.7$ & 6.0 & $4.4 \pm 2.3$ & 0.7 & $85.7 \pm 2.3$ & 89.5 \\
\hline
\end{tabular}

$v=[0,100,0]$, and $v=[0,0,100]$ to define the S1-, S1.8- and

SB-training groups, respectively.

The training method used is the supervised regression training (SupervisedDataSet within PyBrain) with one hidden layer. In this method the training process is carried out until the network reliably matches the "a priori" known optical classification.

\subsection{ANN classification for the full data set}

The ANN training was able to converge to a solution. We then classified all the available spectra in our sample (including those used for the training process).

For each spectrum the ANN gave a set of three elements. Each one of these elements can be considered as an indicator of the resemblance of an X-ray spectrum to the trained X-ray spectra of the S1, S1.8, and SB classes. For example, a spectrum fully consistent with the S1, S1.8, or SB classes should show a vector equal to $(100,0,0),(0,100,0)$, or $(0,0,100)$, respectively. If on the other hand a spectrum is the combination of the $\mathrm{S} 1$, $\mathrm{S} 1.8$ and SB-training sets, we would expect that the sum of $v_{\mathrm{S} 1}$, $v_{\mathrm{S} 1.8}$, and $v_{\mathrm{SB}}$ is equal to 100 (or consistent within errors). The larger the number of $v_{\mathrm{S} 1}, v_{\mathrm{S} 1.8}$, or $v_{\mathrm{SB}}$ the closer the spectrum will resemble the X-ray spectra of the S1-S1.8- or SB-training sets, respectively.

We also assigned errors $(\Delta v)$ to each of these three elements of the ANN for each spectrum, using Monte Carlo simulations. We trained and classified the objects 100 times so that they converge to individual solutions. For each training we obtained 1000 solutions randomly varying the spectra within the measurement error bars for each energy bin. The final solution is the mean value for the 100 thousand runs (i.e., 100 times 1000 solutions) and $\Delta v$ is its standard deviation. Columns 8-10 in Table 1 show the results for $v_{\mathrm{S} 1}, v_{\mathrm{S} 1.8}$, and $v_{\mathrm{SB}}$, respectively.

Values significantly above 100 or below 0 indicate that the spectra cannot be reproduced with the training classes. None of the objects in our class showed ANN components above 100 or below 0 at $\sim 1.5 \sigma$ level). Thus, all of them can be characterised by a combination of the training sets.

The efficiency of the network on the training process can be estimated by its success on classifying the training sets. It has successfully classified 25 out of the 27 spectra within $10 \%$ error (typical error obtained by the ANN). Thus, the efficiency of the network is $290 \%$. Only one S1 (NGC 4639) and two S1.8s (NGC 4168 and NGC 4565) were misclassified, showing $v_{\mathrm{SB}}>10$. However, they show $v_{\mathrm{S} 1}$ and $v_{\mathrm{S} 1.8}$ fully consistent with their training sets within the errors (i.e., S1-training for NGC 4639 and S1.8-training for NGC 4168 and NGC 4565).

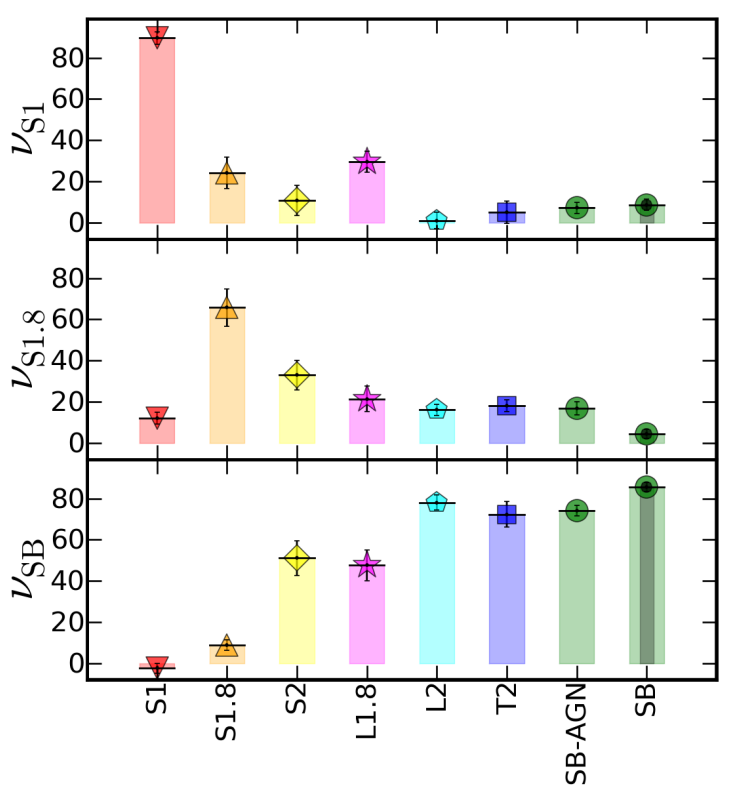

Fig. 1. Histogram of the mean value of the ANN components for each optical class. Error bars represent one sigma over the mean for each distribution. The optical classes are shown as: S1 (upside down red triangles), S1.8 (orange triangles), S2 (yellow diamonds), L1.8 (purple stars), L2 (light-blue pentagons), T2 (dark-blue squares), SB-AGN (green circles), and SB (green circles with small black dots).

\section{Results}

\subsection{Mean value of the ANN components per optical class}

First we present our results regarding the average value of the ANN $\left(\bar{v}_{\mathrm{S} 1}, \bar{v}_{\mathrm{S} 1.8}\right.$, and $\left.\bar{v}_{\mathrm{SB}}\right)$ for each optical class. The mean, its error, and the median values for the ANN components per optical class are shown in Table 2. Figure 1 shows these mean values (and the errors as error bars) as a function of optical classes. The error of the mean of each ANN component is very small in all the optical classes. This indicates that all the sources in each class have similar X-ray spectra. Second, the mean values are not the same in all classes. Therefore, the mean X-ray spectrum is not the same for all of them. We also obtain that

- among the training classes, both $\mathrm{S} 1$ and $\mathrm{S} 1.8$ classes show low $\bar{v}_{\mathrm{SB}}$. The $\mathrm{S} 1$ class shows high $\bar{v}_{\mathrm{S} 1}$ and low $\bar{v}_{\mathrm{S} 1.8}$; the opposite is true for the S1.8 class. Similarly, the SB class shows high $\bar{v}_{\mathrm{SB}}$ and low $\bar{v}_{\mathrm{S} 1}$ and $\bar{v}_{\mathrm{S} 1.8}$. This was expected because we have trained the network to achieve this objective. However, we used all the spectra and not only those used for the training process. Thus, it seems that any eventual flux variations of S1, S1.8, and SB are not associated 


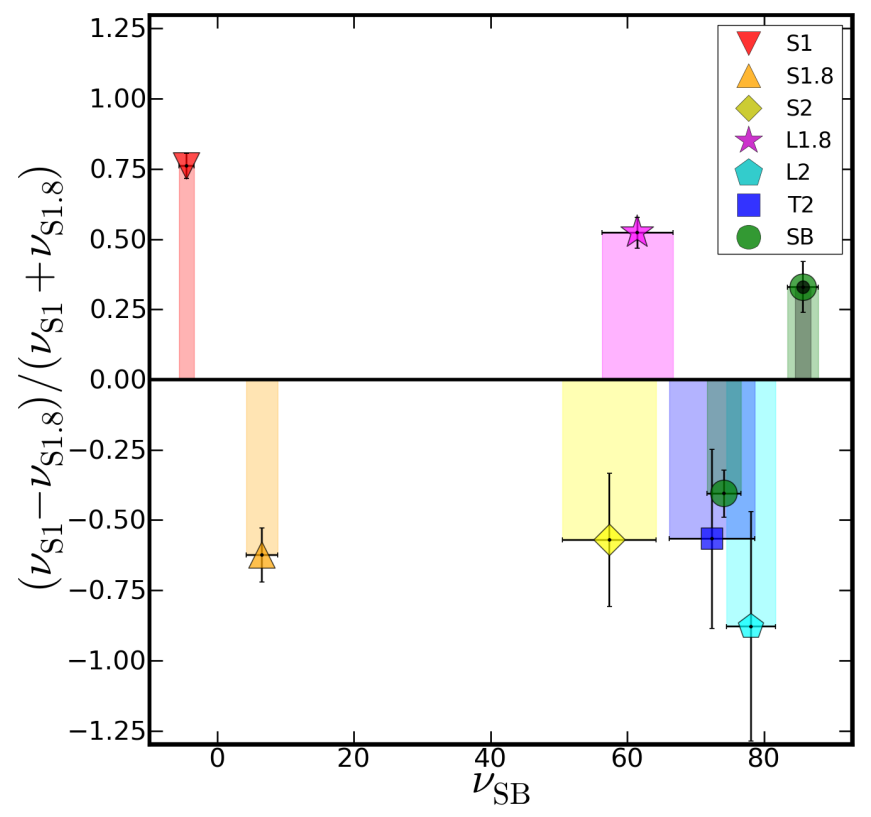

Fig. 2. Ratio of the difference between $v_{\mathrm{S} 1}$ and $v_{\mathrm{S} 1.8}$ over $\left(v_{\mathrm{S} 1}+v_{\mathrm{S} 1.8}\right)$ versus $v_{\mathrm{SB}}$. The optical classes are shown as $\mathrm{S} 1$ (upside down red triangles), S1.8 (orange triangles), S2 (yellow diamonds), L1.8 (purple stars), L2 (light-blue pentagons), T2 (dark-blue squares), SB-AGN (green circles), and SB (green circles with small black dots).

with spectral variations that can dramatically alter the shape of their X-ray spectra.

- the S2, L1.8, L2, T2, and SB-AGN classes are incompatible with any of the trained classes (i.e., S1, S1.8, or SB). They can be interpreted as a combination of two of the three ANN components (see Sect. 5.3).

- the S2 class is inconsistent with the S1 or the S1.8 classes. On average, S2 objects show very low $\bar{v}_{\mathrm{S} 1}$, a $\bar{v}_{\mathrm{S} 1.8}$ that lies between that of the S1 and the S1.8 classes, and $\bar{\nu}_{\mathrm{SB}}$ significantly higher than the respective mean value for the $\mathrm{S} 1$ and S1.8 classes (see Table 2).

- the L1.8 and L2 classes are different. Their $\bar{\nu}_{\mathrm{S} 1.8}$ and $\bar{\nu}_{\mathrm{SB}}$ values are similar to those of the $\mathrm{S} 2$ class. As a class, though, L1.8 can be distinguished from $\mathrm{S} 2$, because their average $\bar{v}_{\mathrm{S} 1}$ is higher $\left(\bar{v}_{\mathrm{S} 1}=30 \pm 5\right)$ than the same value in $\mathrm{S} 2 \mathrm{~s}$ $\left(\bar{v}_{\mathrm{S} 1}=8 \pm 8\right.$, see Table 2$)$.

- the L2, T2, and SB-AGN objects have similar X-ray spectra (see Table 2), although they show different spectral signatures at optical wavelengths.

In summary, our results show that the ANN is able to distinguish six classes of objects based on their X-ray spectral shape: S1, S1.8, S2, L1.8, L2/T2/SB-AGN, and SB. One of the main differences among them is the contribution of the SB-like component, which increases as follows: $\mathrm{S} 1 \Rightarrow \mathrm{S} 1.8 \Rightarrow \mathrm{S} 2 / \mathrm{L} 1.8 \Rightarrow$ $\mathrm{L} 2 / \mathrm{T} 2 / \mathrm{SB}-\mathrm{AGN} \Rightarrow \mathrm{SB}$.

Furthermore, in addition to the Seyfert classes, the L2/T2/SB-AGN X-ray class of objects show a non-zero S1.8 component $\left(\bar{v}_{\mathrm{S} 1.8} \simeq 16\right)$ in their X-ray spectra, while the L1.8 class shows a non-zero $\mathrm{S} 1$ component $\left(\bar{v}_{\mathrm{S} 1} \simeq 30\right)$. Therefore, our results are consistent with the hypothesis that, on average, all emission line nuclei in nearby galaxies host an AGN component, albeit of small strength in many of them.

To better distinguish among the classes, we built the diagram seen in Fig. 2 which shows $\left(v_{\mathrm{S} 1}-v_{\mathrm{S} 1.8}\right) /\left(v_{\mathrm{S} 1}+v_{\mathrm{S} 1.8}\right)$ versus $v_{\mathrm{SB}}$. Positive (negative) values of $\left(v_{\mathrm{S} 1}-v_{\mathrm{S} 1.8}\right) /\left(v_{\mathrm{S} 1}+v_{\mathrm{S} 1.8}\right)$ are expected for classes similar to the $\mathrm{S} 1$ (S1.8) class. The L1.8 class is similar to the $\mathrm{S} 1$ class with higher $\bar{v}_{\mathrm{SB}}$ than the $\mathrm{S} 1$ class. The S2, L2, T2, and SB-AGN classes are like the S1.8 class, but with higher $\bar{v}_{\mathrm{SB}}$ than this class. The S2 class shows $\bar{v}_{\mathrm{SB}}$ similar to that of the L1.8 class. The L2, T2, and SB-AGN classes are indistinguishable. The SB class shows positive values of $\left(v_{\mathrm{S} 1}-v_{\mathrm{S} 1.8}\right) /\left(v_{\mathrm{S} 1}+v_{\mathrm{S} 1.8}\right)$ with the highest $\bar{v}_{\mathrm{SB}}$ among the optical classes.

\subsection{ANN component plane}

All the objects in our sample are described by a combination of the three vectors of the ANN, whose sum in most cases is close to 100 including the error bars (i.e., $v_{\mathrm{S} 1}+v_{\mathrm{S} 1.8}+v_{\mathrm{SB}} \simeq 100$ ). Taking advantage of this, Fig. 3 shows the diagram of the ANN components, plotted on a plane with these axes. The corners of the triangle represent the locus for the S1-training, S1.8-training, and SB-training classes. The lines connecting each pair of these points indicate the locus on this plane for which the third component is zero.

The ANN plane is not uniformly filled. Instead, objects tend to occupy specific areas of this plane, which are distinctive of each class. This is another way to show that the X-ray spectral shape for objects in a particular class is similar in all of them, and at the same time, that these X-ray spectra are different among the various optical classes.

Most objects in the S1 and S1.8 classes are spread along a line that connects the S1- and S1.8-training locus (except NGC 1068 and NGC 1275). The SB-class of objects occupies the lower right part of the diagram, close to the SB-training locus. The objects in the L2 and T2 classes also occupy the same part of the diagram. Thus, the X-ray spectra of L2, T2, and SB-AGN are similar and close to the pure SB class (already mentioned in the previous section). Objects in the L1.8 class are spread along the line that connects the S1- and the SB-training locus. Objects belonging to the $\mathrm{S} 2$ class are spread along the line connecting the S1.8- and SB-training locus.

\subsection{Correlations for the ANN components}

Motivated by the results reported in the previous section regarding the position of the objects in each class in the ANN plane, we investigated the correlations between pairs of the ANN parameters. In this way, we basically projected the ANN plane onto the $v_{\mathrm{S} 1.8}-v_{\mathrm{S} 1}, v_{\mathrm{SB}}-v_{\mathrm{S} 1.8}$, and $v_{\mathrm{SB}}-v_{\mathrm{S} 1}$ relations.

Figure 4 shows $v_{\mathrm{S} 1.8}$ versus $v_{\mathrm{S} 1}$ (top row), $v_{\mathrm{SB}}$ versus $v_{\mathrm{S} 1.8}$ (middle row), and $v_{\mathrm{SB}}$ versus $v_{\mathrm{S} 1}$ (bottom row). The dashed lines in each plot indicate the locus of points for which the sum of the two ANN components is equal to 100. If an object lies on this line, then its spectrum can be reproduced by a combination of only the two ANN components relevant for each plot. For example, the X-ray spectra of the objects that are located on the diagonal line of the $v_{\mathrm{S} 1.8}$ versus $v_{\mathrm{S} 1}$ plot should be reproduced by a combination of only the S1.8 and S1 average X-ray spectra. Likewise for the objects located close to the dashed lines of the other panels.

For each object in our sample, we computed the distance of each pair of its ANN components from the respective diagonal line and placed this object in the panel where this distance has the lowest value. The first main result from Fig. 4 is that most objects in our sample are located very close (i.e., within the errors) to a diagonal line in one of the panels of this figure. This implies that the X-ray spectra in our sample are fully consistent with a combination of only two ANN components.

Most of the objects belonging to the S1 and S1.8 classes are located in the top-left panel in Fig. 4. Moreover, instead of 


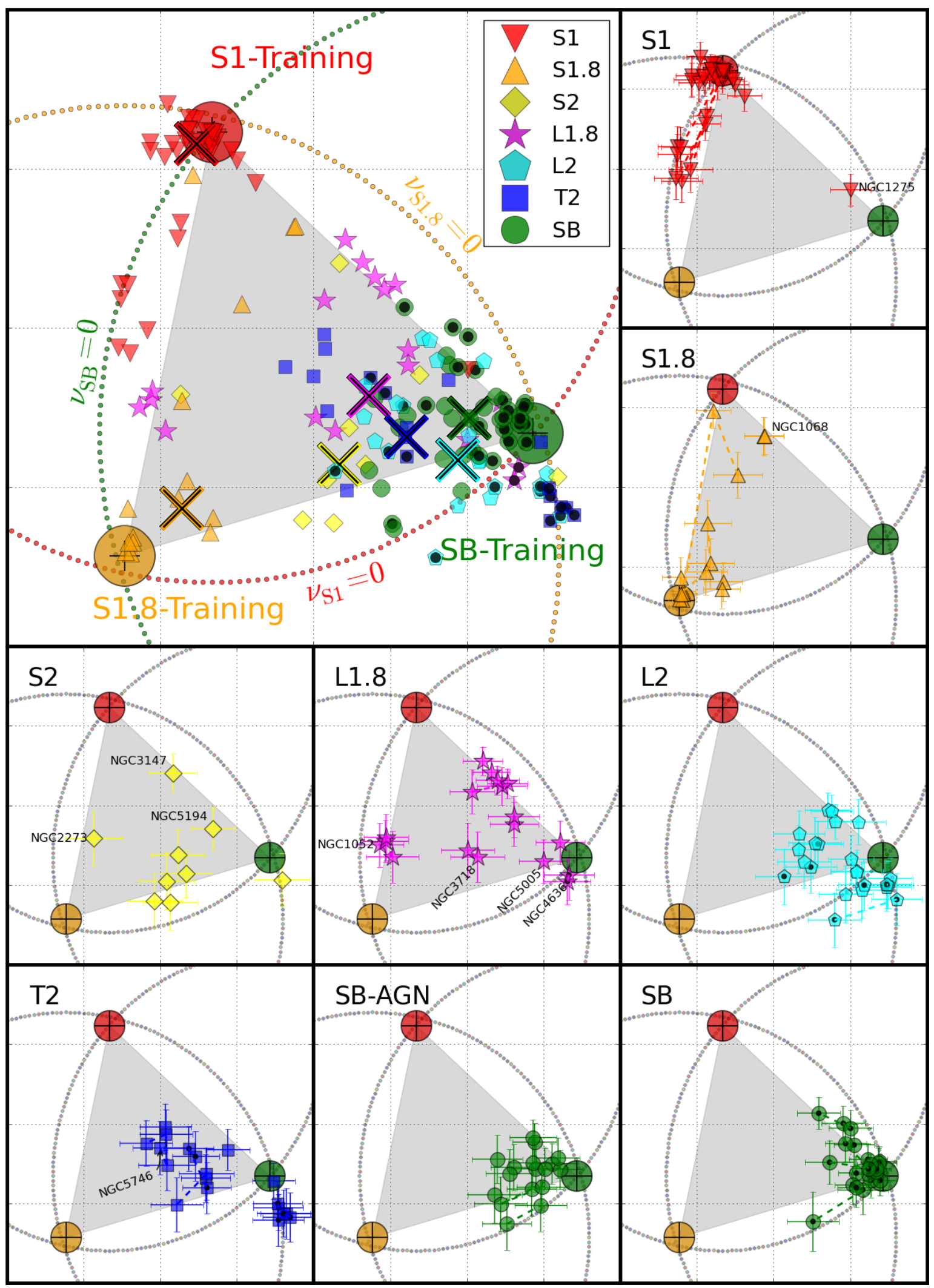

Fig. 3. Diagram of the ANN results. The corners of the triangle show the locus expected for S1-training (large red circle), S1.8-training (large orange circle), and SB-training (large green circle). The red, orange, and green dotted circles (centred on the corners of the triangles) correspond to $v_{\mathrm{S} 1}=0, v_{\mathrm{S} 1.8}=0$, and $v_{\mathrm{SB}}=0$. The optical classes are shown as $\mathrm{S} 1$ (upside down red triangles), $\mathrm{S} 1.8$ (orange triangles), $\mathrm{S} 2$ (yellow diamonds), L1.8 (purple stars), L2 (light-blue pentagons), T2 (dark-blue squares), SB-AGN (green circles), and SB (green circles with small black dots). Black dots indicate objects that might not be AGN according to the literature (see Table 1). Large crosses represent the mean locus for each optical class. The smaller panels show the diagrams for each optical class. Dashed lines connect observations of the same source. We have marked the names of the relevant objects for Sects. 5.2 and 5.3. 

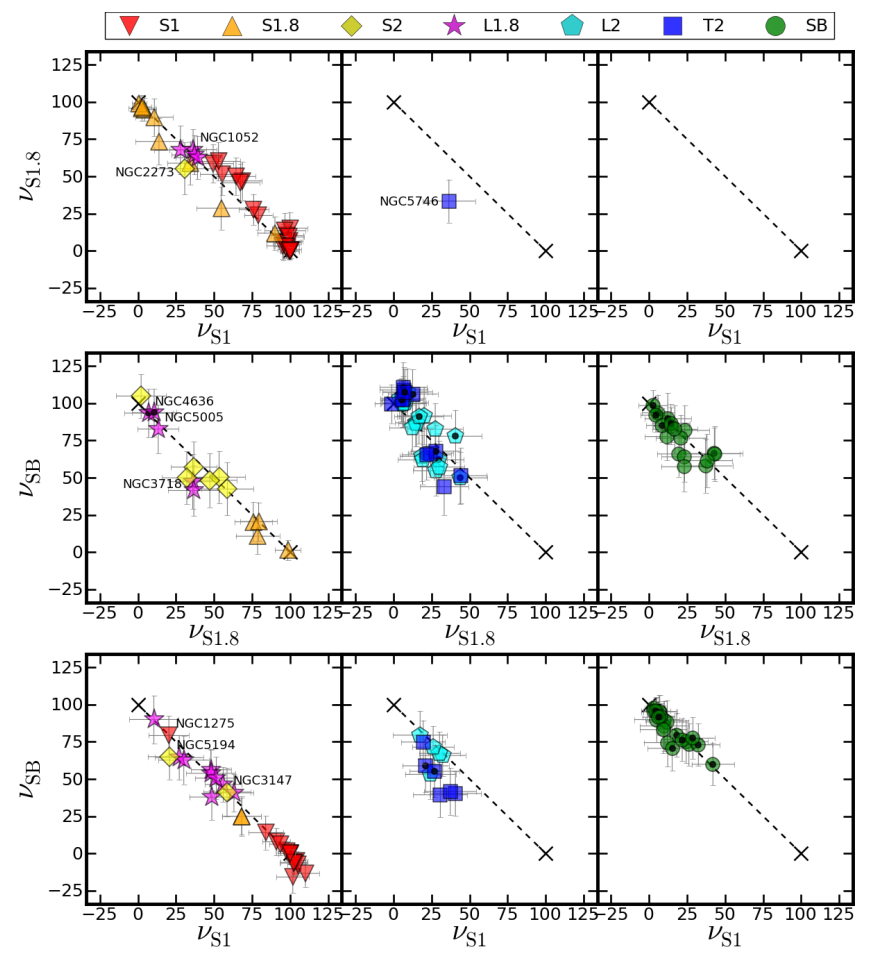

Fig. 4. ANN components $v_{\mathrm{S} 1.8}$ versus $v_{S 1}$ (top row), $v_{\mathrm{S} B}$ versus $v_{\mathrm{S} 1.8}$ (middle row), and $v_{\mathrm{S} B}$ versus $v_{\mathrm{S} 1}$ (bottom row). Each row is split into three panels for the S1, S1.8, S2, and L1.8 classes (left), L2 and T2 classes (middle) and SB class (right). The dashed line shows the expected locus if the component not involved in the plot is negligible. Each plot shows objects that are closer to its dashed line than to the dashed line of the other two plots. The optical classes are shown as S1 (upside down red triangles), S1.8 (orange triangles), S2 (yellow diamonds), L1.8 (purple stars), L2 (light-blue pentagons), T2 (dark-blue squares), SB-AGN (green circles), and SB (green circles with small black dots). Black dots indicate objects that might not be AGN according to the literature (see Table 1).

being located around the $v_{\mathrm{S} 1}$ or $v_{\mathrm{S} 1.8}$, they show a continuous range of values along this diagonal line. Among the other optical classes, only NGC 1052 and NGC 2273 are placed in the same locus. Thus, these two sources, despite the optical classification, behave at X-rays as the S1 and S1.8 classes in our sample.

There are no other objects, from any class, located in the $v_{\mathrm{S} 1.8}-v_{\mathrm{S} 1}$ diagonal line, except NGC 5746. Although this source was classified as T2 by Ho et al. (1997), our results indicate that its X-ray spectrum is very similar to that of S1 and S1.8 objects. Apart from this exception, the X-ray spectra of all emission line nuclei other than S1 and S1.8 classes show the contribution of a component that does not appear in the $\mathrm{S} 1$ and $\mathrm{S} 1.8$ classes.

Most of the objects belonging to the S2 class are located along the line that connects $v_{\mathrm{SB}}$ and $v_{\mathrm{S} 1.8}$ with, on average $v_{\mathrm{SB}}<60$ and little contribution from $v_{\mathrm{S} 1}$ (except NGC 2273, NGC 5194 and NGC 3147, Fig. 4, middle row, left panel). Thus, they are similar to the $\mathrm{S} 1.8$ class, but with higher contributions of the $v_{\mathrm{SB}}$ component.

Most of the objects belonging to the L1.8 class fall in the $v_{\mathrm{SB}}$ versus $v_{\mathrm{S} 1}$ line (except NGC 1052, NGC 3718, NGC 4636, and NGC 5005). L2 and T2 classes are placed in the same locus in these diagrams. Thus, according to the ANN, L2 and $\mathrm{T} 2$ classes belong to the same category. Most of them are closer to the line that connects $v_{\mathrm{SB}}$ and $v_{\mathrm{S} 1.8}$ (Fig. 4, middle row, middle panel), although some of them are located along the line that connects $v_{\mathrm{SB}}$ and $v_{\mathrm{S} 1}$ (Fig. 4, bottom row, middle panel). Moreover, a few spectra of these $\mathrm{T} 2$ objects are those located at a slightly larger distance from the diagonal line, although still consistent with it. SB-AGN also seem to be located along the line connecting $v_{\mathrm{SB}}$ and $v_{\mathrm{S} 1.8}$ (Fig. 4, middle row, right panel). Finally, most of the SB objects are located in the diagonal line connecting $v_{\mathrm{SB}}$ and $v_{\mathrm{S} 1}$ (Fig. 4, bottom row, right panel).

Based on the fact that most of the X-ray spectra in our sample can be regarded as a combination of two ANN components, we present the following scheme for the classification, based on their average $\mathrm{X}$-ray spectra:

- S1 and S1.8: they show no $v_{\mathrm{SB}}$ component $\left(\bar{v}_{\mathrm{SB}}=-1.7 \pm 1.2\right.$ for $\mathrm{S} 1$ and $\mathrm{S} 1.8$ classes together). High values of the $v_{\mathrm{S} 1}$ component are found for the $\mathrm{S} 1$ class and high values of the $v_{\mathrm{S} 1.8}$ component for the S1.8 class (see Table 2). The X-ray spectra of the objects in these two classes show a mixture of the $v_{\mathrm{S} 1}$ and $v_{\mathrm{S} 1.8}$ components with a wide range of values (see Fig. 4, top-left panel).

- S2: they show negligible $v_{\mathrm{S} 1}$ within the one-sigma deviation. Their X-ray spectra are a combination of the $v_{\mathrm{SB}}$ and the $v_{\mathrm{S} 1.8}$ components.

- L1.8: the contribution of the $v_{\mathrm{SB}}$ component resembles that of the S2 class (see Table 2). However, they show higher contribution of the $v_{\mathrm{S} 1}$ component than the $\mathrm{S} 2$ class.

- L2/T2/SB-AGN: this family of objects shows almost no $v_{\mathrm{S} 1}$ component $\left(\bar{v}_{\mathrm{S} 1}=4.1 \pm 2.5\right)$, a strong $v_{\mathrm{SB}}$ component $\left(\bar{v}_{\mathrm{SB}}=75.1 \pm 2.6\right)$, and a weaker $v_{\mathrm{S} 1.8}$ component $\left(\bar{v}_{\mathrm{S} 1.8}=17.0 \pm 1.6\right)$. It can be distinguished from the $\mathrm{S} 2$ class because of their significantly higher mean value of the $v_{\mathrm{SB}}$ component (see Table 2).

- SB: this is the class of objects that show the highest values for the $v_{\mathrm{SB}}$ component $\left(\bar{v}_{\mathrm{SB}}=86 \pm 2\right)$ and almost non-existent $v_{\mathrm{S} 1}\left(\bar{v}_{\mathrm{S} 1}=8.7 \pm 2.7\right)$ and $v_{\mathrm{S} 1.8}$ components $\left(\bar{v}_{\mathrm{S} 1.8}=4.4 \pm 2.3\right)$.

\section{Discussion}

We have shown that the ANN analysis can be useful to classify the main optical classes using only X-ray spectra. In general, an object with $v_{\mathrm{SB}} \leq 10$ is almost certainly an $\mathrm{S} 1$ or $\mathrm{S} 1.8$. Moreover, an object with low $v_{\mathrm{S} 1.8}$ and high $v_{\mathrm{S} 1}$ and $v_{\mathrm{SB}}$ is most probably an L1.8, while an object with low $v_{\mathrm{S} 1}$ and high $\nu_{\mathrm{S} 1.8}$ and $\nu_{\mathrm{SB}}$ is most probably an $\mathrm{S} 2$. A higher percentage of $v_{\mathrm{SB}}$ characterises the L2, T2 and SB nuclei. However, we would like to stress that most of the differences are found when we consider the average value for each class. Thus, although the ANN method is very useful to study the average properties, it may not be as successful in classifying a single object based on its ANN components. Using the results regarding the average properties of the objects in each class, in this section we discuss the following questions: (1) type 1/type 2 dichotomy; (2) optical versus X-ray classes; and (3) elusive AGN. Finally, we present the usefulness of this analysis for its application to X-ray surveys.

\subsection{Type $1 /$ type 2 dichotomy}

Our results indicate that the X-ray spectra of the S1 and $\mathrm{S} 1.8$ classes can be reproduced by a mixture of the $v_{\mathrm{S} 1}$ and $v_{\mathrm{S} 1.8}$ components, with $v_{\mathrm{S} 1}$ and $v_{\mathrm{S} 1.8}$ being stronger in the former and latter classes, respectively. Furthermore, the S1 and S1.8 classes show a continuous range of values of the $v_{\mathrm{S} 1}$ and $v_{\mathrm{S} 1.8}$ components (see Fig. 4, top-left panel). Our analysis cannot offer direct indications of the nature of the $v_{\mathrm{S} 1}$ or the $v_{\mathrm{S} 1.8}$ components, or for the physical parameter that drives their correlation 


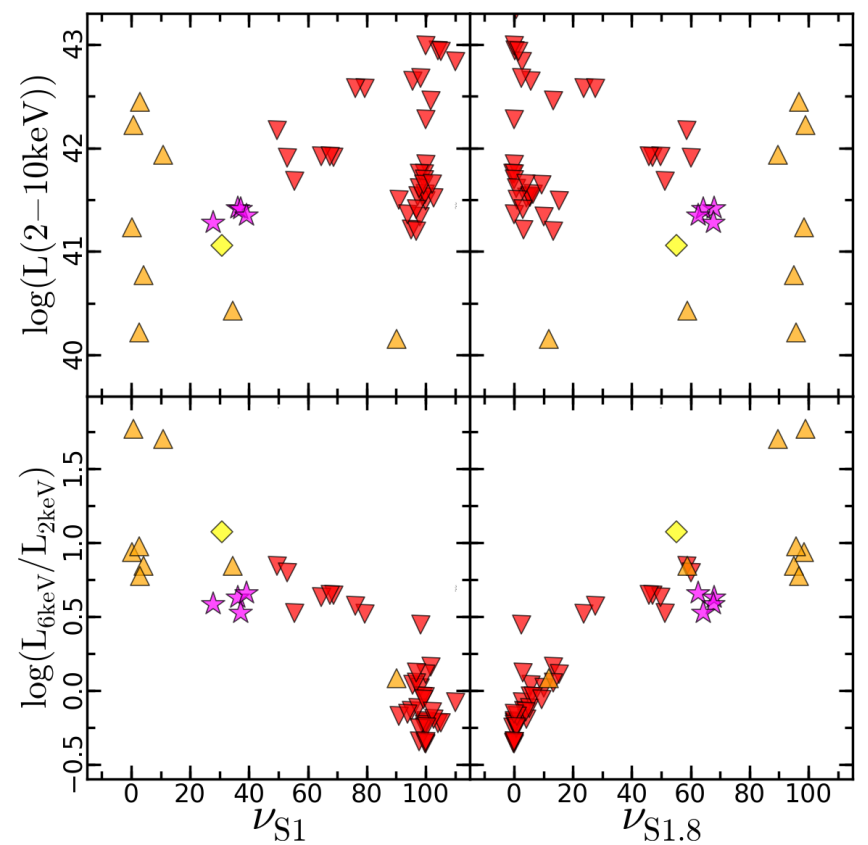

Fig. 5. Top: logarithmic of the $2-10 \mathrm{keV}$ band observed luminosity, $\log (L(2-10 \mathrm{keV}))$, versus the $v_{\mathrm{S} 1}$ (left) and $v_{\mathrm{S} 1.8}$ (right) components. Bottom: logarithmic of the ratio between the observed luminosity at 6 $\mathrm{keV}$ versus the observed luminosity at $2 \mathrm{keV}, \log \left(L_{6 \mathrm{keV}} / L_{2} \mathrm{keV}\right)$, versus the $v_{\mathrm{S} 1}$ (left) and $v_{\mathrm{S} 1.8}$ (right) components. We only plot objects with $v_{\mathrm{SB}}<10$ (see text). The optical classes are shown as: $\mathrm{S} 1$ (upside down red triangles), S1.8 (orange triangles), S2 (yellow diamonds), and L1.8 (purple stars).

for S1s and S1.8s. Below we discuss possible interpretations of this result.

The continuous range of values for $v_{\mathrm{S} 1}$ and $v_{\mathrm{S} 1.8}$ could reflect a continuous range of absorptions (i.e., $N_{\mathrm{H}}$ ), increasing for the S1.8 class. This is consistent with the UM of AGN. Indeed, $\mathrm{X}$-rays have been used in AGN to study the amount of absorption (Risaliti et al. 1999; Bianchi et al. 2012; Ho 2008). Risaliti et al. (1999) found that $75 \%$ of their type 2 Seyferts were heavily obscured $\left(N_{\mathrm{H}}>10^{23} \mathrm{~cm}^{-2}\right), 50 \%$ of them were Compton-thick (i.e., $N_{\mathrm{H}}>1.5 \times 10^{24} \mathrm{~cm}^{-2}$ ), with the $\mathrm{S} 1.8$ class characterised by an average lower $N_{\mathrm{H}}$ than the $\mathrm{S} 2$ class. Alternatively, a low flux level continuum was recently suggested by Elitzur et al. (2014) as the main reason to classify objects as S1.8s. They suggested that intermediate types of objects are part of an evolutionary sequence where the BLR slowly disappears as the bolometric luminosity decreases. Hence, the continuous range of values for $v_{\mathrm{S} 1}$ and $v_{\mathrm{S} 1.8}$ might be interpreted either as (1) an increase of the absorption as we move from S1s and S1.8s, or (2) a decrease of the AGN continuum flux in S1.8s. As shown below, our results favour the first interpretation.

Assuming that $L(2-10 \mathrm{keV})$ is an indication of the total luminosity, we would expect it to be proportional to $v_{\mathrm{S} 1}$ and inversely correlated with $v_{\mathrm{S} 1.8}$ if a decrease of the intrinsic continuum is responsible for the S1.8 class. Figure 5 (top panels) shows the $\log (L(2-10 \mathrm{keV}))^{4}$ versus $v_{\mathrm{S} 1}$ (left) and $v_{\mathrm{S} 1.8}$ (right) for objects with a negligible contribution of $v_{\mathrm{SB}}\left(v_{\mathrm{SB}}<10\right)$. At each $v_{\mathrm{S} 1}$ or $v_{\mathrm{S} 1.8}$ values there is a large scatter of luminosities, but objects with high (low) $v_{\mathrm{S} 1}\left(v_{\mathrm{S} 1.8}\right)$ have higher X-ray luminosities, on average. The Pearson correlation coefficients are $r=0.37$

\footnotetext{
$4 L(2-10 \mathrm{keV})$ is computed as the sum of all the bins in the calibrated spectra in the $2-10 \mathrm{keV}$ band multiplied by the size of the spectral bin $(\Delta E=0.05 \mathrm{keV})$.
}

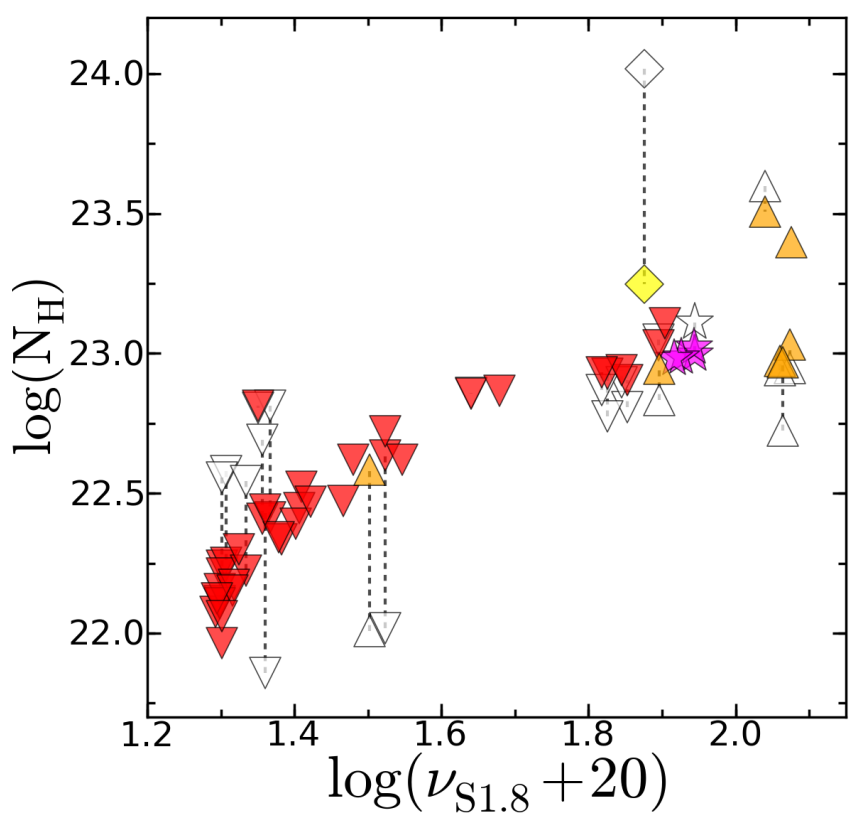

Fig. 6. Logarithmic of $N_{\mathrm{H}}$ versus $\log \left(v_{\mathrm{S} 1.8}+20\right)$. Filled symbols show $N_{\mathrm{H}}$ values using a simple power-law model to the $2-10 \mathrm{keV}$ band (see Appendix A). Empty symbols show $N_{\mathrm{H}}$ reported in the literature when available (see Table A.1). Dashed vertical lines link the $N_{\mathrm{H}}$ values using a simple power-law model and those reported in the literature.

$\left(P_{\text {null }}=0.008\right)$ and $r=0.34\left(P_{\text {null }}=0.015\right)$ for the correlations with $v_{\mathrm{S} 1}$ and $v_{\mathrm{S} 1.8}$, respectively (see Fig. 5, top, right and left panels). The small numbers of the correlation coefficients shows that the correlations are weak, although the null hypothesis probability indicates that it may be significant.

The bottom panels of Fig. 5 show the steepness of the spectra, expressed as $\log \left(L_{6 \mathrm{keV}} / L_{2} \mathrm{keV}\right)^{5}$, versus the $v_{\mathrm{S} 1}$ (left) and $v_{\mathrm{S} 1.8}$ (right) components. The X-ray spectra become harder (i.e., the emission at $6 \mathrm{keV}$ becomes more prominent than the emission at $2 \mathrm{keV}$ ) when the $v_{\mathrm{S} 1.8}$ component increases (and $v_{\mathrm{S} 1}$ decreases). The correlation between them shows Pearson correlation coefficients and null probabilities of $r=0.91$, $P_{\text {null }}=6 \times 10^{-20}$ and $r=0.89, P_{\text {null }}=7 \times 10^{-18}$, respectively.

Irrespective of the reason for the spectral hardening, the strength of the correlations in the lower panels of Fig. 5 indicates that the distributions of the $v_{\mathrm{S} 1}$ and $v_{\mathrm{S} 1.8}$ components in Seyferts are not driven primarily by luminosity, but by the spectral hardening of their X-ray spectra. The simplest explanation for this spectral hardening is an increase of absorption, which in the case of Compton-thin sources affects the $2 \mathrm{keV}$ flux much stronger than the $6 \mathrm{keV}$ flux. Therefore, based on the strength of the correlations shown in Fig. 5, it seems reasonable to assume that a variable amount of obscuration is the main physical parameter responsible for the continuous range of $v_{\mathrm{S} 1}$ and $v_{\mathrm{S} 1.8}$. The same effect can also explain the weak correlations with luminosity (see Fig. 5, top panels). If the observed luminosities were corrected for absorption, then both S1.8 and S1 might show the same level of X-ray luminosity. Therefore, the scenario to be preferred is that in which obscuration is responsible for the type 1/type 2 dichotomy. This is fully consistent with the UM of AGN, in which the obscuring torus is responsible for blocking the inner parts of the AGN (both the BLR and the X-ray source) in type 2 galaxies.

\footnotetext{
$5 L_{2} \mathrm{keV}$ and $L_{6} \mathrm{keV}$ are the monochromatic luminosities at $2 \mathrm{keV}$ and $6 \mathrm{keV}$, respectively, obtained from the flux-calibrated spectra.
} 
A final check on the nature of this dichotomy can be performed by comparing $v_{\mathrm{S} 1.8}$ with the absorbing column density, $N_{\mathrm{H}}$, for these observations (see Fig. 6 and Appendix A for details on the measurements of $\left.N_{\mathrm{H}}\right)$. The quantity $\log \left(\nu_{\mathrm{S} 1.8}+20\right)^{6}$ is linearly related with $\log \left(N_{\mathrm{H}}\right)\left(r=0.93, P_{\text {null }}=1.5 \times 10^{-21}\right)$ when derived with a simple power-law fit (filled symbols in Fig. 6). A less significant linear relation $\left(r=0.57, P_{\text {null }}=5.3 \times 10^{-3}\right)$ is found when using $N_{\mathrm{H}}$ estimates reported in the literature (empty symbols in Fig. 6). This weaker relationship is probably due to (1) fewer observations with $N_{\mathrm{H}}$ and (2) different models used for the spectral fittings for each observation. It reinforces the importance of a self-consistent modelling for the sample to compare the parameters.

\subsection{Optical versus $X$-ray classes}

The ANN has found differences on the average X-ray spectra of the six different classes: S1, S1.8, S2, L1.8, L2/T2/SBAGN, and SB. Thus, the L2, T2, and SB-AGN belong to the same X-ray category according to the ANN results. Division lines in the BPT diagrams were developed and adapted as a function of the ionisation models and/or observations available (e.g., Veilleux \& Osterbrock 1987; Osterbrock 1989; Kewley et al. 2001, 2006, 2013; Kauffmann et al. 2003; Stasińska et al. 2006). Objects close to the division between star-forming galaxies and AGN could be classified as L2, T2, or SB depending on how these divisions are set and/or how these three diagrams are used together. This could explain why the L2, T2, and SB-AGN classes cannot be distinguished at X-rays according to the ANN. Alternatively, the number of physical parameters governing the classes at X-rays might be lower than those driving the optical classes.

One of the main differences between the X-ray spectra of the various optical classes is set by the $v_{\mathrm{SB}}$ component, which increases from the $\mathrm{S} 1$ to the $\mathrm{SB}$ classes, passing through the $\mathrm{S} 1.8$, $\mathrm{S} 2, \mathrm{~L} 1.8$, and L2/T2/SB-AGN groups. The nature of the $v_{\mathrm{SB}}$ component cannot be fully assessed with the results of this analysis alone, but we discuss possible explanations below.

The star-formation (circumnuclear or that of the host galaxy) is the most natural explanation for the $v_{\mathrm{SB}}$ component. In this case, X-ray emission by binary systems, supernovae remnants, and/or emission by diffuse hot gas might contribute to this $v_{\mathrm{SB}}$ component. In this case, we would expect $\bar{v}_{\mathrm{SB}}$ to increase when the luminosity decreases for the objects in our sample. To test this hypothesis, Fig. 7 shows the average $v_{\mathrm{SB}}\left(\bar{v}_{\mathrm{SB}}\right)$ versus the mean value for $\log (L(2-10 \mathrm{keV}))$. These two quantities are clearly anti-correlated $\left(r=0.94, P_{\text {null }}=5 \times 10^{-5}\right)^{7}$. Thus, $v_{\mathrm{SB}}$ increases when the X-ray luminosity decreases, which supports our hypothesis that the $v_{\mathrm{SB}}$ component is related to star formation. The SB galaxies, with the highest $v_{\mathrm{SB}}$ values in our sample, have X-ray luminosities of $\sim 10^{40} \mathrm{erg} \mathrm{s}^{-1}$. This could be representative of the galactic X-ray emission from the processes mentioned above. If an AGN component is present in almost all galaxies, then as it becomes stronger, $v_{\mathrm{SB}}$ decreases, while at the same time the X-ray luminosity increases. The $v_{\mathrm{SB}}$ component is almost zero in the $\mathrm{S} 1.8$ and $\mathrm{S} 1$ classes probably because the AGN-like source entirely outshines the underlying host-galaxy emission, or it could also mean that the $v_{\mathrm{SB}}$ component is entirely absent. For example, Wu et al. (2009, and references therein)

\footnotetext{
6 Note that we computed the logarithmic of $\left(v_{\mathrm{S} 1.8}+20\right)$ to avoid negative values of $v_{\mathrm{S} 1.8}$.

7 Note that $v_{\mathrm{S} 1}$ and $v_{\mathrm{S} 1.8}$ show a poor relation with $\log (\bar{L}(2-10 \mathrm{keV}))$ in the top panel of Fig. 5.
}

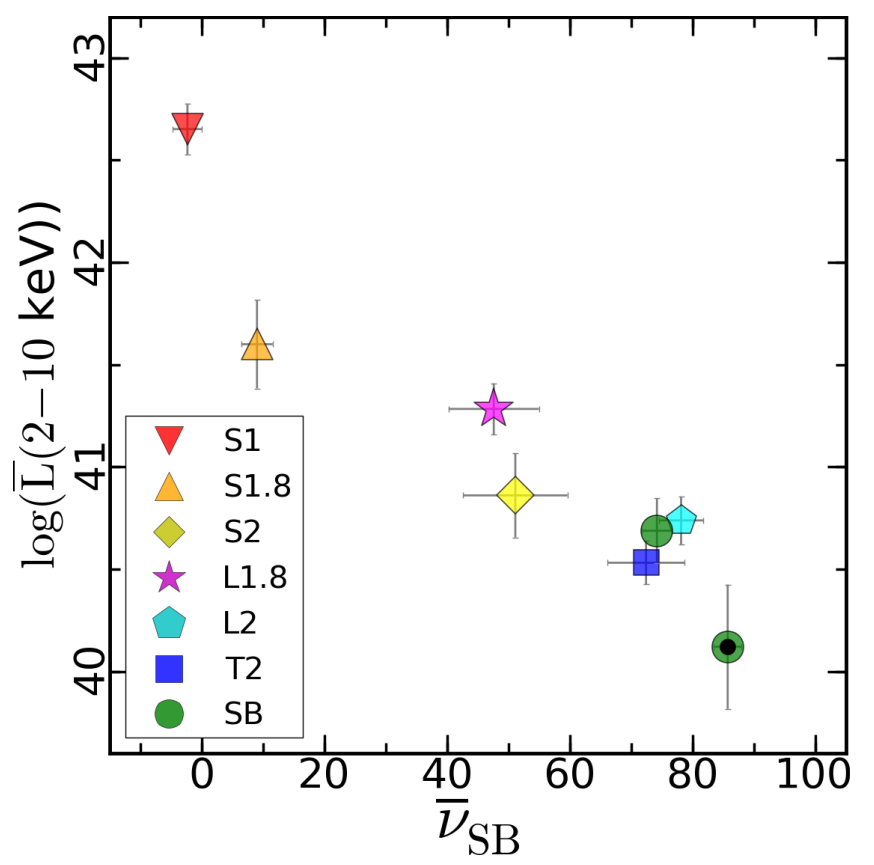

Fig. 7. Mean $\bar{v}_{\mathrm{SB}}$ component versus mean $2-10 \mathrm{keV}$ band luminosity observed in logarithmic scale, $\log (\bar{L}(2-10 \mathrm{keV}))$, per optical class. The optical classes are shown as S1 (upside down red triangles), S1.8 (orange triangles), S2 (yellow diamonds), L1.8 (purple stars), L2 (lightblue pentagons), T2 (dark-blue squares), SB-AGN (green circles), and $\mathrm{SB}$ (green circles with small black dots).

claimed that the circumnuclear star formation might be even destroyed in the presence of an AGN.

An alternative origin for the $v_{\mathrm{SB}}$ component for sources hosting an AGN is the X-ray emission from the hot plasma in the NLR, emission from the scattering component in AGN or ionised gas. It has been claimed that high-resolution X-ray spectra are dominated by emission lines from the NLR in type 2 Seyferts (Guainazzi \& Bianchi 2007). Moreover, the soft X-ray emission in a few AGN is extended on scales ranging from a few hundred parsecs to a few thousand parsecs, in close agreement with the morphology of the NLR seen at optical wavelengths for both LINERs and type 2 Seyferts (González-Martín et al. 2010; Bianchi et al. 2006; Masegosa et al. 2011). In this case, we would expect $\bar{v}_{\mathrm{SB}}$ to increase when the luminosity increases for the objects in our sample. However, as mentioned before, $v_{\mathrm{SB}}$ increases when the X-ray luminosity decreases (see Fig. 7), which rules out the NLR as the main responsible factor for the $v_{\mathrm{SB}}$ component.

\subsection{Elusive AGN}

The $v_{\mathrm{S} 1}$ and/or $v_{\mathrm{S} 1.8}$ components are significant in most of the emission line nuclei presented in this paper (see Figs. 1 and 4). A total of 22 out of the 162 spectra (i.e., 13.5\%) are consistent with no signature of an AGN-like component; this percentage is slightly higher in terms of the number of objects (19 out of the $90,21 \%$ ). Thus, $\sim 80 \%$ of our sample show signs of an AGN-like component, either with an S1-like or an S1.8-like contribution. This number is almost twice the percentage of AGN (43\%) estimated at optical frequencies by Ho et al. (1997) for the same sample. Moreover, although for some T2, SB-AGN, and SB nuclei, the $v_{\mathrm{S} 1}$ and $v_{\mathrm{S} 1.8}$ components are consistent with zero, on average, the X-ray spectra of these nuclei do show the 
presence of $\nu_{\mathrm{S} 1}$ or $\nu_{\mathrm{S} 1.8}$ components. However, based on their optical spectra, these classes correspond, at best, to objects on the border between AGN and star-forming galaxies.

Our result strongly supports the hypothesis that an AGN component might be present at X-rays at a certain level in most of the emission line nuclei included in our sample, even if they do not show signatures of this AGN component in their optical spectra. Non-AGN at optical wavelengths with AGN signatures at X-rays have often been studied in the literature (called elusive AGN, see Maiolino et al. 1998; Soria et al. 2006a,b). Galaxies with bulges harbour BHs (see Kormendy \& Ho 2013, and references therein). However, at optical wavelengths, only a small fraction of bulge galaxies show evidence for AGN activity; in about half of the high S/N optical spectra taken by Ho et al. (1997) there is no indication of AGN activity. Tzanavaris \& Georgantopoulos (2007) studied a sample of star-forming galaxies classified by Ho et al. (1997) at X-ray, finding AGN signatures for a large part of them. This is consistent with our results. Tzanavaris \& Georgantopoulos (2007) suggested that the lack of optical signatures may arise because the emission could be overwhelmed by that coming from circumnuclear star formation. This is entirely consistent with the increase of the $v_{\mathrm{SB}}$ component when the luminosity decreases (see Fig. 7 and previous Section), if the $v_{\mathrm{SB}}$ component is associated with the constant, diffuse X-ray emission of the host galaxy and/or X-ray emission associated with intense star-forming regions.

\subsection{Relevance of the ANN method for X-ray surveys}

Artificial neural networks have proven to be a powerful approach to a broad variety of problems (e.g., Bishop 1996; Gupta et al. 2004; Asensio Ramos \& Socas-Navarro 2005; Socas-Navarro 2005; Carballo et al. 2008; Han \& Han 2012). In the most common application, ANN functions as a classification algorithm. In the AGN field, for instance, Rawson et al. (1996) already used the ANN to classify optical spectra into type 1 and type 2 AGN. However, ANN have not been used to classify X-ray spectra before.

Using other statistical methods, several attempts have been made to classify X-ray spectra, particularly for low $\mathrm{S} / \mathrm{N}$ spectra. Norman et al. (2004) selected normal, type 1 and type 2 AGN galaxies from the Chandra Deep field North (CDF-N) and South (CDF-S) samples using a Bayesian classification procedure. Priors were constructed from a set of galaxies with welldefined optical classes. They used the X-ray hardness ratio, the $0.5-2 \mathrm{keV} \mathrm{X}$-ray luminosity, and the ratio between X-ray and optical fluxes. The product of the prior distribution for a class and the likelihood for the observed parameters for a given source gave the probability that the source was drawn from that class. Ptak et al. (2007) used a similar methodology with several improvements (e.g., $k$-correction in the optical data). They showed that the method was efficient in classifying the X-ray spectra into type 1 , type 2 and normal galaxies. Our methodology has two advantages: (1) it does not need any optical information and (2) it is able to distinguish among the S1, S1.8, L1.8, S2, L2/T2/SB-AGN, and SB classes. We show that the ANN is an excellent tool to distinguish between most of the optical classes using only their X-ray spectra. It might be very useful for X-ray surveys where the optical information is lacking. The ANN components can be computed for any set of X-ray spectra using our already trained $\mathrm{ANN}^{8}$. The effects of using X-ray spectra with lower $\mathrm{S} / \mathrm{N}$ to their classification with the ANN method needs to be explored (perhaps through simulations), which is beyond the scope of this paper. Finally, the ANN should be able to classify objects in broad classes, and the results will be useful for statistical studies. However, the method is not particularly useful in the classification of objects on an individual basis.

\section{Summary}

We have investigated the connection between optical classes and X-ray spectra in a sample of 90 nearby emission line galaxies. We used flux-calibrated X-ray spectra observed with $X M M-N e w t o n / \mathrm{pn}$. The results of this paper are, for the first time, free of the subjectivity of the X-ray spectral fitting thanks to the use of the ANNs:

- We used a set of the S1, S1.8 and SB classes to train the ANN, giving as output arrays $v_{\mathrm{S} 1}, v_{\mathrm{S} 1.8}$, and $v_{\mathrm{SB}}$, respectively. The ANN is $90 \%$ efficient to distinguish these classes. They all show distinctive signatures at X-rays.

- Based on their X-ray spectral shape, the emission line nuclei in the nearby galaxies were divided into six groups: $\mathrm{S} 1$, S1.8, S2, L1.8, L2/T2/SB-AGN, and SB classes. Only the L2, T2, and SB-AGN classes show the same average X-ray spectrum, even though they belong to distinct optical classes. Furthermore, the objects within each of these six classes have similar average X-ray spectra.

- The average X-ray spectrum of the objects in each X-ray class can be described by the contribution of two components, either the $v_{\mathrm{SB}}$ and $v_{\mathrm{S} 1}$, the $v_{\mathrm{SB}}$ and $v_{\mathrm{S} 1.8}$, or the $v_{\mathrm{S} 1}$ and $v_{\mathrm{S} 1.8}$ (in the case of S1s and S1.8s). The S2 (L1.8) class is similar to the $\mathrm{S} 1.8$ ( $\mathrm{S} 1$ ) class, but with higher contributions of the $v_{\mathrm{SB}}$ component. The L2/T2 and SB-AGN classes have a strong $v_{\mathrm{SB}}$ component, with the addition of a $v_{\mathrm{S} 1.8}$ component.

- The S1 and S1.8 classes show low $v_{\mathrm{SB}}$ and a wide range of the $v_{\mathrm{S} 1}$ and $v_{\mathrm{S} 1.8}$ components. We showed that this wide range of $v_{\mathrm{S} 1}$ and $v_{\mathrm{S} 1.8}$ contributions is most probably related to the different amount of obscuration that affects the nuclear emission at X-rays, in agreement with the UM predictions.

- Most of the objects in our sample have a significant contribution of either a $v_{\mathrm{S} 1}$ or a $v_{\mathrm{S} 1.8}$ component. This result strongly supports the presence of an AGN-like nucleus in most nearby galaxies, albeit at different levels of luminosities (i.e. activity).

- We argued that the $v_{\mathrm{SB}}$ component is associated to a contribution of star-formation in the host galaxy. As the contribution of the AGN component decreases, the $v_{\mathrm{SB}}$ component increases, and at optical wavelengths it shows stronger signatures representative of S2, L1.8, L2/T2/SB-AGN, and finally of SB nuclei.

We find that the emission line nuclei in nearby galaxies can be classified into six classes, based on the shape of their X-ray spectra. These classes are associated with the traditional optical classes, although there are fewer of them. Thus, the shape of the X-ray spectra of those galaxies may be determined by fewer physical parameters than those that determine the optical classes. Alternatively, this could be due to the difficulties to classify them at optical wavelength using the BPT diagrams. Our results suggest that the X-ray spectra of nearby galaxies are simply the

\footnotetext{
8 We kindly suggest to contact any of the coauthors of the paper for the use of our trained ANN.
} 
combination of two components. The first one is an AGN-like component, the second one is due to star formation in the host galaxy that contributes to the X-rays. An AGN-like nucleus may be present in most of them $(80 \%)$. Its strength, relative to the contribution of star-formation in the host-galaxy, determines the average X-ray spectrum of objects for each X-ray class. A third physical parameter might be related to the amount of obscuring material along the LOS. This parameter almost certainly drives the type 1/type 2 dichotomy, but may also explain why, for example, the L1.8 class predominantly shows a $v_{\mathrm{S} 1}$ component in their spectra while L2, T2, and SB-AGN predominantly show a $v_{\mathrm{S} 1.8}$ component.

We conclude that the ANN method is quite powerful to detect AGN-like nuclei (and distinguish which ones are affected by absorption). It can therefore be used to identify AGN, and even to infer their optical classes, using only X-ray spectra and our trained ANN. However, this can only be done in a statistical way, that is, using the X-ray spectra of many objects. This methodology may be very useful in X-ray surveys, for example the eRosita survey, where the optical information for tens of thousands of newly discovered objects will not be available.

Acknowledgements. We thank the anonymous referee for his/her useful comments and suggestions. The authors acknowledge the Spanish MINECO through project Consolider-Ingenio 2010 Program grant CSD2006-00070: First Science with the GTC (http://www.iac.es/consolider-ingenio-gtc/) and AYA2012-39168C03-01. This work was also partially funded by the Spanish MINECO through a Juan de la Cierva Fellowship. This work was financed by MINECO grant AYA 2010-15169, Junta de Andalucía TIC114 and Proyecto de Excelencia de la Junta de Andalucía P08-TIC-03531. L.H.G. also acknowledges grant BES-2011043319. Based on observations obtained with XMM-Newton, an ESA science mission with instruments and contributions directly funded by ESA Member States and NASA. This research has made use of data obtained from the High Energy Astrophysics Science Archive Research Center (HEASARC), provided by NASA's Goddard Space Flight Center.

\section{References}

Akylas, A., \& Georgantopoulos, I. 2008, A\&A, 479, 735

Akylas, A., \& Georgantopoulos, I. 2009, A\&A, 500, 999

Alonso-Herrero, A., Ramos Almeida, C., Mason, R., et al. 2011, ApJ, 736, 82

Antonucci, R. 1993, ARA\&A, 31, 473

Antonucci, R. R. J., \& Miller, J. S. 1985, ApJ, 297, 621

Asensio Ramos, A., \& Socas-Navarro, H. 2005, A\&A, 438, 1021

Baldwin, J. A., Phillips, M. M., \& Terlevich, R. 1981, PASP, 93, 5

Ballet, J. 1999, A\&AS, 135, 371

Ballet, J. 2001, Astronomical Data Analysis Software and Systems X, eds. F. R.

Harnden, Jr., Francis A. Primini, \& H. E. Payne, ASP Conf. Proc., 238, 381

Bianchi, S., Guainazzi, M., \& Chiaberge, M. 2006, A\&A, 448, 499

Bianchi, S., Maiolino, R., \& Risaliti, G. 2012, Adv. Astron., 2012,

Bishop, C.M. 1996, Neural networks for pattern recognition (Oxford University Press)

Brightman, M., \& Nandra, K. 2011, MNRAS, 413, 1206

Cappi, M., Panessa, F., Bassani, L., et al. 2006, A\&A, 446, 459

Carballo, R., González-Serrano, J. I., Benn, C. R., \& Jiménez-Luján, F. 2008, MNRAS, 391, 369

Cid Fernandes, R., González Delgado, R. M., Schmitt, H., et al. 2004, ApJ, 605, 105

Comastri, A. 2004, Supermassive Black Holes in the Distant Universe, Astrophys. Space Sci. Lib. (Kluwer Academic Publishers), 308, 245

Davis, T. A., Bureau, M., Cappellari, M., Sarzi, M., \& Blitz, L. 2013, Nature, 494, 328

Dopita, M. A., \& Sutherland, R. S. 1995, ApJ, 455, 468

Elitzur, M., \& Ho, L. C. 2009, ApJ, 701, L91
Elitzur, M., Ho, L. C., \& Trump, J. R. 2014, MNRAS, 438, 3340

Elmegreen, D. M., Chromey, F. R., \& Santos, M. 1998, AJ, 116, 1221

González Delgado, R. M., Pérez, E., Cid Fernandes, R., \& Schmitt, H. 2008, AJ, 135,747

González-Martín, O., Masegosa, J., Márquez, I., Guerrero, M. A., \& DultzinHacyan, D. 2006, A\&A, 460, 45

González-Martín, O., Masegosa, J., Márquez, I., Guainazzi, M., \& JiménezBailón, E. 2009a, A\&A, 506, 1107

González-Martín, O., Masegosa, J., Márquez, I., \& Guainazzi, M. 2009b, ApJ, 704, 1570

González-Martín, O., Acosta-Pulido, J. A., Perez Garcia, A. M., \& Ramos Almeida, C. 2010, ApJ, 723, 1748

González-Martín, O., Papadakis, I., Braito, V., et al. 2011, A\&A, 527, A142

Goulding, A. D., \& Alexander, D. M. 2009, MNRAS, 398, 1165

Goulding, A. D., Alexander, D. M., Bauer, F. E., et al. 2012, ApJ, 755, 5

Guainazzi, M., \& Bianchi, S. 2007, MNRAS, 374, 1290

Gupta, R., Singh, H. P., Volk, K., \& Kwok, S. 2004, ApJS, 152, 201

Heckman, T. M. 1980, A\&A, 87, 152

Hernández-García, L., González-Martín, O., Márquez, I., \& Masegosa, J. 2013, A\&A, 556, A47

Han, Y., \& Han, Z. 2012, ApJ, 749, 123

Ho, L. C. 2008, ARA\&A, 46, 475

Ho, L. C., Filippenko, A. V., \& Sargent, W. L. W. 1997, ApJ, 487, 568

Kauffmann, G., Heckman, T. M., Tremonti, C., et al. 2003, MNRAS, 346, 1055

Kewley, L. J., Heisler, C. A., Dopita, M. A., \& Lumsden, S. 2001, ApJS, 132, 37

Kewley, L. J., Groves, B., Kauffmann, G., \& Heckman, T. 2006, MNRAS, 372, 961

Kewley, L. J., Maier, C., Yabe, K., et al. 2013, ApJ, 774, L10

Kormendy, J., \& Ho, L. C. 2013, ARA\&A, 51, 511

Maiolino, R., Salvati, M., Bassani, L., et al. 1998, A\&A, 338, 781

Masegosa, J., Márquez, I., Ramirez, A., \& González-Martín, O. 2011, A\&A, 527, A 23

Moustakas, J., Kennicutt, R. C., Jr., Tremonti, C. A., et al. 2010, ApJS, 190, 233

Nicholson, K. L., Mittaz, J. P. D., \& Mason, K. O. 1997, MNRAS, 285, 831

Nikołajuk, M., \& Walter, R. 2013, A\&A, 552, A75

Norman, C., Ptak, A., Hornschemeier, A., et al. 2004, ApJ, 607, 721

Osterbrock, D. E. 1989, Astrophysics of Gaseous Nebulae and Active Galactic Nuclei (Mill Valley, CA: University Science Books)

Panessa, F., Bassani, L., Cappi, M., et al. 2006, A\&A, 455, 173

Ptak, A., Mobasher, B., Hornschemeier, A., Bauer, F., \& Norman, C. 2007, ApJ, 667,826

Randall, S. W., Jones, C., Kraft, R., Forman, W. R., \& O’Sullivan, E. 2009, ApJ, 696, 1431

Ramos Almeida, C., Levenson, N. A., Alonso-Herrero, A., et al. 2011, ApJ, 731, 92

Rawson, D. M., Bailey, J., \& Francis, P. J. 1996, PASA, 13, 207

Risaliti, G., Maiolino, R., \& Salvati, M. 1999, ApJ, 522, 157

Sanders, J. S., Fabian, A. C., \& Dunn, R. J. H. 2005, MNRAS, 360, 133

Schaul, T., Bayer, J., Wierstra, D., et al. 2010, J. Mach. Learn. Res., 11, 743

Socas-Navarro, H. 2005, ApJ, 621, 545

Soria, R., Fabbiano, G., Graham, A. W., et al. 2006a, ApJ, 640, 126

Soria, R., Graham, A. W., Fabbiano, G., et al. 2006b, ApJ, 640, 143

Stasińska, G., Cid Fernandes, R., Mateus, A., Sodré, L., \& Asari, N. V. 2006, MNRAS, 371, 972

Strüder, L., Briel, U., Dennerl, K., et al. 2001, A\&A, 365, L18

Terlevich, R., \& Melnick, J. 1985, MNRAS, 213, 841

Tombesi, F., Cappi, M., Reeves, J. N., et al. 2010, A\&A, 521, A57

Tomita, A., Aoki, K., Watanabe, M., Takata, T., \& Ichikawa, S.-i. 2000, AJ, 120, 123

Tran, H. D. 2001, ApJ, 554, L19

Tran, H. D. 2003, ApJ, 583, 632

Tristram, K. R. W., Meisenheimer, K., Jaffe, W., et al. 2007, A\&A, 474, 837

Tzanavaris, P., \& Georgantopoulos, I. 2007, A\&A, 468, 129

Urry, C. M., \& Padovani, P. 1995, PASP, 107, 803

Veilleux, S., \& Osterbrock, D. E. 1987, ApJS, 63, 295

Véron-Cetty, M.-P., \& Véron, P. 2006, A\&A, 455, 773

Wu, Y., Charmandaris, V., Huang, J., Spinoglio, L., \& Tommasin, S. 2009, ApJ, 701,658

Younes, G., Porquet, D., Sabra, B., \& Reeves, J. N. 2011, A\&A, 530, A149

Pages 12 to 23 are available in the electronic edition of the journal at http://www . aanda.org 
Table 1. Sample properties and results.

\begin{tabular}{|c|c|c|c|c|c|c|c|c|c|c|}
\hline \multirow[b]{2}{*}{$\begin{array}{r}\text { Num } \\
(1)\end{array}$} & \multirow[b]{2}{*}{$\begin{array}{l}\text { Name } \\
\text { (2) }\end{array}$} & \multirow[b]{2}{*}{$\begin{array}{l}\text { ObsID } \\
\text { (3) }\end{array}$} & \multirow[b]{2}{*}{$\begin{array}{c}\text { Class } \\
\text { (4) }\end{array}$} & \multirow[b]{2}{*}{$\begin{array}{c}\text { Expos. } \\
\text { (5) }\end{array}$} & \multirow[b]{2}{*}{$\begin{array}{r}\text { Counts } \\
(6)\end{array}$} & \multicolumn{4}{|c|}{ ANN } & \multirow{2}{*}{$\begin{array}{r}\text { Comments } \\
\text { (11) }\end{array}$} \\
\hline & & & & & & $\begin{array}{c}\text { Train } \\
\text { (7) }\end{array}$ & $\begin{array}{l}v_{\mathrm{S} 1} \\
(8)\end{array}$ & $\begin{array}{r}v_{\mathrm{S} 1.8} \\
(9)\end{array}$ & $\begin{array}{r}v_{\mathrm{SB}} \\
(10)\end{array}$ & \\
\hline 1 & IC 10 & 152260101 & $\mathrm{H}$ & 30 & 15238 & - & $18.2 \pm 13.3$ & $-2.7 \pm 13.1$ & $79.5 \pm 14.7$ & \\
\hline \multirow[t]{3}{*}{2} & IC 342 & 093640901 & $\mathrm{H}$ & 4 & 1283 & TR & $-0.6 \pm 11.2$ & $5.9 \pm 10.7$ & $93.6 \pm 12.2$ & \\
\hline & & 206890201 & & 16 & 3108 & - & $-5.3 \pm 16.6$ & $12.3 \pm 15.3$ & $89.6 \pm 17.3$ & \\
\hline & & 206890401 & & 3 & 744 & - & $-14.2 \pm 16.9$ & $43.0 \pm 18.7$ & $66.2 \pm 17.4$ & \\
\hline 3 & NGC 315 & 305290201 & L1.9 & 13 & 5785 & - & $10.5 \pm 16.0$ & $0.0 \pm 13.5$ & $89.9 \pm 16.1$ & AGN (1) \\
\hline \multirow[t]{2}{*}{4} & NGC 410 & 203610201 & $\mathrm{~T} 2:$ & 13 & 5848 & - & $-25.3 \pm 16.7$ & $6.7 \pm 15.8$ & $108.9 \pm 15.2$ & Non-AGN (1) \\
\hline & & 304160201 & & 5 & 2400 & - & $-26.6 \pm 16.7$ & $12.6 \pm 16.4$ & $106.2 \pm 16.5$ & \\
\hline \multirow[t]{4}{*}{5} & NGC 598 & 141980501 & $\mathrm{H}$ & 1 & 9053 & - & $9.9 \pm 12.4$ & $-1.3 \pm 10.3$ & $89.5 \pm 12.6$ & \\
\hline & & 141980801 & & 7 & 34989 & TR & $3.0 \pm 5.6$ & $-0.3 \pm 4.2$ & $97.4 \pm 5.5$ & \\
\hline & & 102640101 & & 5 & 33527 & - & $7.1 \pm 10.8$ & $-4.9 \pm 9.6$ & $95.2 \pm 11.1$ & \\
\hline & & 102642101 & & 8 & 25042 & - & $6.6 \pm 11.8$ & $-3.9 \pm 9.4$ & $95.3 \pm 11.1$ & \\
\hline \multirow[t]{2}{*}{6} & NGC 777 & 304160301 & $\mathrm{~S} 2 / \mathrm{L}$ & 0 & 639 & - & $-27.8 \pm 17.3$ & $40.9 \pm 17.2$ & $77.9 \pm 17.6$ & Non-AGN * \\
\hline & & 203610301 & & 3 & 3138 & - & $-27.4 \pm 16.5$ & $9.2 \pm 16.4$ & $107.6 \pm 15.8$ & \\
\hline \multirow[t]{4}{*}{7} & NGC 1052 & 553300401 & L1.9 & 46 & 30645 & - & $36.3 \pm 15.6$ & $67.9 \pm 13.8$ & $-4.3 \pm 8.3$ & AGN (1) \\
\hline & & 093630101 & & 11 & 5954 & - & $27.9 \pm 17.1$ & $67.7 \pm 16.2$ & $3.4 \pm 12.4$ & \\
\hline & & 553300301 & & 42 & 27269 & - & $37.2 \pm 15.1$ & $64.4 \pm 13.2$ & $-1.7 \pm 8.2$ & \\
\hline & & 306230101 & & 44 & 25545 & - & $39.2 \pm 14.5$ & $62.5 \pm 14.6$ & $-1.8 \pm 9.4$ & \\
\hline \multirow[t]{2}{*}{8} & NGC 1068 & 111200101 & S1.8 & 32 & 368177 & - & $68.3 \pm 12.7$ & $2.7 \pm 12.1$ & $24.9 \pm 12.3$ & CT (2) \\
\hline & & 111200201 & & 27 & 316879 & - & $68.2 \pm 12.9$ & $2.2 \pm 11.7$ & $25.0 \pm 13.2$ & \\
\hline 9 & NGC 1275 & 085110101 & S1.5 & 22 & 431300 & - & $20.3 \pm 13.2$ & $-0.6 \pm 10.0$ & $79.2 \pm 13.3$ & \\
\hline 10 & NGC 1569 & 112290801 & $\mathrm{H}$ & 11 & 2178 & $\mathrm{TR}$ & $-2.1 \pm 10.2$ & $2.8 \pm 9.5$ & $98.4 \pm 10.2$ & \\
\hline 11 & NGC 2146 & 110930101 & $\mathrm{H}$ & 6 & 3430 & - & $10.0 \pm 16.1$ & $4.0 \pm 12.7$ & $85.7 \pm 16.8$ & AGN (3) \\
\hline 12 & NGC 2273 & 140951001 & S2 & 3 & 455 & - & $30.8 \pm 18.7$ & $55.2 \pm 17.5$ & $0.9 \pm 18.1$ & CT $(2,4)$ \\
\hline 13 & NGC 2342 & 093190501 & $\mathrm{H}$ & 23 & 2532 & TR & $3.0 \pm 11.4$ & $4.5 \pm 10.0$ & $92.0 \pm 11.3$ & \\
\hline 14 & NGC 2655 & 301650301 & $\mathrm{~S} 2$ & 1 & 590 & - & $-10.2 \pm 17.7$ & $53.4 \pm 17.1$ & $50.3 \pm 17.5$ & AGN (1) \\
\hline 15 & NGC 2787 & 200250101 & L1.9 & 25 & 2511 & - & $27.2 \pm 15.8$ & $10.3 \pm 13.9$ & $64.3 \pm 15.0$ & AGN (1) \\
\hline 16 & NGC 2841 & 201440101 & $\mathrm{~L} 2$ & 9 & 1731 & - & $16.8 \pm 15.5$ & $28.1 \pm 15.2$ & $54.7 \pm 16.7$ & AGN (1) \\
\hline 17 & NGC 2903 & 556280301 & $\mathrm{H}$ & 54 & 15114 & TR & $3.1 \pm 6.9$ & $0.6 \pm 5.8$ & $95.7 \pm 7.1$ & \\
\hline 18 & NGC 3079 & 110930201 & $\mathrm{~S} 2$ & 4 & 1132 & - & $3.9 \pm 16.3$ & $36.8 \pm 15.0$ & $57.1 \pm 17.1$ & CT $(2,4)$ \\
\hline 19 & NGC 3147 & 405020601 & $\mathrm{~S} 2$ & 12 & 8073 & - & $58.6 \pm 15.4$ & $2.9 \pm 12.2$ & $41.2 \pm 16.2$ & True-S2 (5) \\
\hline \multirow[t]{2}{*}{20} & NGC 3226 & 101040301 & L1.9 & 30 & 6518 & - & $48.6 \pm 14.7$ & $12.3 \pm 13.7$ & $37.8 \pm 15.4$ & AGN (1) \\
\hline & & 400270101 & & 93 & 28221 & - & $48.1 \pm 13.2$ & $-3.7 \pm 9.5$ & $56.2 \pm 13.4$ & \\
\hline 21 & NGC 3227 & 101040301 & S1.5 & 30 & 32042 & - & $55.4 \pm 14.4$ & $51.2 \pm 14.0$ & $-6.0 \pm 8.2$ & \\
\hline & & 400270101 & & 93 & 1184930 & TR & $100.0 \pm 2.5$ & $0.0 \pm 2.1$ & $-0.0 \pm 2.5$ & \\
\hline 22 & NGC 3310 & 556280101 & $\mathrm{H}$ & 21 & 15781 & - & $24.4 \pm 14.3$ & $2.4 \pm 11.7$ & $75.1 \pm 14.4$ & AGN (3) \\
\hline & & 556280201 & & 24 & 16355 & - & $26.2 \pm 15.2$ & $0.8 \pm 11.2$ & $73.1 \pm 14.2$ & \\
\hline 23 & NGC 3367 & 551450101 & $\mathrm{H}$ & 9 & 1572 & - & $9.7 \pm 16.0$ & $11.9 \pm 14.6$ & $77.7 \pm 16.0$ & AGN (6) \\
\hline 24 & NGC 3516 & 107460601 & $\mathrm{~S} 1.2$ & 43 & 249895 & - & $95.6 \pm 11.0$ & $5.7 \pm 12.0$ & $-1.9 \pm 10.1$ & \\
\hline & & 107460701 & & 81 & 299978 & - & $101.8 \pm 10.7$ & $13.4 \pm 12.5$ & $-15.9 \pm 10.8$ & \\
\hline & & 401210501 & & 40 & 1112450 & - & $104.2 \pm 5.9$ & $0.3 \pm 5.5$ & $-4.9 \pm 6.3$ & \\
\hline & & 401210601 & & 42 & 589580 & - & $110.1 \pm 9.2$ & $2.7 \pm 9.4$ & $-13.4 \pm 9.1$ & \\
\hline & & 401210401 & & 30 & 880682 & TR & $100.0 \pm 2.4$ & $0.0 \pm 2.1$ & $-0.0 \pm 2.5$ & \\
\hline & & 401211001 & & 35 & 933142 & - & $105.1 \pm 6.6$ & $1.6 \pm 5.9$ & $-7.3 \pm 7.0$ & \\
\hline 25 & NGC 3623 & 082140301 & L2: & 25 & 2873 & - & $16.7 \pm 16.5$ & $18.8 \pm 14.6$ & $64.3 \pm 16.7$ & Non-AGN (1) \\
\hline 26 & NGC 3628 & 110980101 & $\mathrm{~T} 2$ & 37 & 6061 & - & $40.7 \pm 16.9$ & $19.3 \pm 14.7$ & $40.2 \pm 15.1$ & AGN (7) \\
\hline 27 & NGC 3665 & 052140201 & $\mathrm{H}:$ & 20 & 2777 & - & $13.8 \pm 15.1$ & $19.7 \pm 14.3$ & $66.0 \pm 15.3$ & AGN (8) \\
\hline 28 & NGC 3690A & 679381101 & $\mathrm{H}$ & 6 & 4058 & - & $5.1 \pm 14.8$ & $3.0 \pm 12.1$ & $89.9 \pm 15.1$ & CT $(2,4)$ \\
\hline & & 112810101 & & 13 & 8136 & - & $12.0 \pm 14.1$ & $-0.4 \pm 12.8$ & $88.3 \pm 14.6$ & \\
\hline 29 & NGC 3718 & 200430501 & L1.9 & 9 & 2479 & - & $14.5 \pm 17.6$ & $36.0 \pm 15.8$ & $47.3 \pm 18.3$ & AGN (1) \\
\hline & & 200431301 & & 7 & 1696 & - & $19.3 \pm 17.3$ & $36.8 \pm 16.9$ & $41.4 \pm 17.2$ & \\
\hline 30 & NGC 3884 & 301900601 & L1.9 & 18 & 7065 & - & $47.9 \pm 16.1$ & $-0.4 \pm 11.7$ & $53.8 \pm 16.4$ & AGN (1) \\
\hline 31 & NGC 3998 & 090020101 & L1.9 & 5 & 35635 & - & $56.1 \pm 12.1$ & $-2.2 \pm 8.7$ & $46.3 \pm 11.7$ & AGN (1) \\
\hline 32 & NGC 4051 & 157560101 & $\mathrm{~S} 1.2$ & 40 & 233043 & - & $95.2 \pm 10.8$ & $3.3 \pm 8.9$ & $0.3 \pm 10.4$ & \\
\hline & & 606321601 & & 24 & 850091 & TR & $100.0 \pm 2.2$ & $0.0 \pm 2.0$ & $0.0 \pm 2.2$ & \\
\hline & & 606320201 & & 16 & 360147 & - & $99.4 \pm 7.1$ & $-0.4 \pm 5.9$ & $-0.2 \pm 7.4$ & \\
\hline
\end{tabular}

Notes. Net number of counts in the X-ray $0.2-10 \mathrm{keV}$ band. Exposure time in ks. Non-AGN marked as \& are those without indications of AGN activity reported in the literature.

References. References for the AGN nature for unconfirmed AGN (AGN/Non-AGN), Compton-thickness (CT), and True Type-2 Seyferts (True,S2) included in Col. 11 (see Section 2): (1) González-Martín et al. (2009a), (2) Goulding et al. (2012), (3) Tzanavaris \& Georgantopoulos (2007), (4) Comastri (2004), (5) Akylas \& Georgantopoulos (2008), (6) Véron-Cetty \& Véron (2006), (7) Goulding \& Alexander (2009), (9) González-Martín et al. (2011), (10) Moustakas et al. (2010), (11) González-Martín et al. (2009b), (12) Davis et al. (2013), (13) Nikołajuk \& Walter (2013), (14) Tomita et al. (2000), (15) Nicholson et al. (1997), (16) Elmegreen et al. (1998), and (17) Randall et al. (2009). 
Table 1. continued.

\begin{tabular}{|c|c|c|c|c|c|c|c|c|c|c|}
\hline \multirow[b]{2}{*}{$\begin{array}{r}\text { Num } \\
(1)\end{array}$} & \multirow[b]{2}{*}{$\begin{array}{l}\text { Name } \\
(2)\end{array}$} & \multirow[b]{2}{*}{$\begin{array}{r}\text { ObsID } \\
(3)\end{array}$} & \multirow[b]{2}{*}{$\begin{array}{r}\text { Class } \\
(4)\end{array}$} & \multirow[b]{2}{*}{$\begin{array}{r}\text { Expos. } \\
(5)\end{array}$} & \multirow[b]{2}{*}{$\begin{array}{r}\text { Counts } \\
(6) \\
\end{array}$} & \multicolumn{4}{|c|}{ ANN } & \multirow{2}{*}{$\begin{array}{r}\text { Comments } \\
\text { (11) }\end{array}$} \\
\hline & & & & & & $\begin{array}{r}\text { Train } \\
(7)\end{array}$ & $\begin{array}{l}v_{\mathrm{S} 1} \\
(8)\end{array}$ & $\begin{array}{r}v_{\mathrm{S} 1.8} \\
(9)\end{array}$ & $\begin{array}{r}v_{\mathrm{SB}} \\
(10)\end{array}$ & \\
\hline & & 606321901 & & 13 & 80791 & - & $98.0 \pm 10.8$ & $10.2 \pm 10.4$ & $-8.5 \pm 11.0$ & \\
\hline & & 606322001 & & 8 & 79259 & - & $98.9 \pm 8.7$ & $5.5 \pm 8.7$ & $-4.6 \pm 9.6$ & \\
\hline & & 606320301 & & 13 & 335449 & - & $97.8 \pm 5.8$ & $-0.1 \pm 5.7$ & $1.3 \pm 6.4$ & \\
\hline & & 606320401 & & 12 & 78135 & - & $99.9 \pm 11.6$ & $15.2 \pm 10.8$ & $-15.4 \pm 11.7$ & \\
\hline & & 606321501 & & 10 & 227170 & - & $99.5 \pm 6.0$ & $-0.1 \pm 5.7$ & $-1.2 \pm 6.8$ & \\
\hline & & 606321701 & & 21 & 183454 & - & $102.7 \pm 8.8$ & $4.1 \pm 7.1$ & $-6.2 \pm 8.8$ & \\
\hline & & 606322301 & & 22 & 317693 & - & $97.2 \pm 8.4$ & $5.2 \pm 8.5$ & $-3.1 \pm 8.1$ & \\
\hline & & 606322201 & & 19 & 192988 & - & $99.9 \pm 8.6$ & $6.4 \pm 8.6$ & $-6.7 \pm 9.7$ & \\
\hline & & 606321401 & & 24 & 454259 & - & $97.9 \pm 5.8$ & $0.8 \pm 5.7$ & $-0.0 \pm 6.4$ & \\
\hline & & 606322101 & & 16 & 62470 & - & $96.7 \pm 13.4$ & $13.4 \pm 12.0$ & $-11.9 \pm 13.5$ & \\
\hline & & 606321801 & & 11 & 129550 & - & $99.2 \pm 10.2$ & $9.3 \pm 8.8$ & $-10.3 \pm 11.5$ & \\
\hline & & 606320101 & & 27 & 319242 & - & $102.2 \pm 8.7$ & $3.9 \pm 6.7$ & $-6.5 \pm 8.8$ & \\
\hline 33 & NGC 4102 & 601780701 & $\mathrm{H}$ & 5 & 1293 & - & $1.7 \pm 17.7$ & $37.1 \pm 17.8$ & $57.9 \pm 18.1$ & AGN (9) \\
\hline 34 & NGC 4138 & 112551201 & S1.9 & 8 & 4253 & TR & $0.2 \pm 8.9$ & $98.5 \pm 8.5$ & $1.2 \pm 6.7$ & \\
\hline 35 & NGC 4143 & 150010601 & L1.9 & 9 & 3153 & - & $30.2 \pm 16.8$ & $6.9 \pm 12.9$ & $62.7 \pm 16.2$ & AGN (1) \\
\hline \multirow{8}{*}{36} & NGC 4151 & 112310101 & S1.5 & 20 & 138931 & - & $68.7 \pm 12.9$ & $46.9 \pm 12.7$ & $-15.4 \pm 8.9$ & \\
\hline & & 112830501 & & 17 & 115289 & - & $64.5 \pm 12.9$ & $49.9 \pm 12.5$ & $-15.2 \pm 8.8$ & \\
\hline & & 112830201 & & 50 & 330369 & - & $67.5 \pm 12.3$ & $45.7 \pm 12.7$ & $-14.3 \pm 9.1$ & \\
\hline & & 143500301 & & 12 & 381987 & TR & $98.2 \pm 4.7$ & $2.4 \pm 4.8$ & $-0.0 \pm 2.2$ & \\
\hline & & 402660201 & & 21 & 197054 & - & $49.5 \pm 13.4$ & $58.6 \pm 12.9$ & $-10.4 \pm 7.7$ & \\
\hline & & 143500201 & & 12 & 294178 & - & $79.2 \pm 9.3$ & $23.7 \pm 9.6$ & $-3.7 \pm 5.0$ & \\
\hline & & 143500101 & & 9 & 232041 & - & $76.1 \pm 9.7$ & $27.7 \pm 10.3$ & $-3.1 \pm 5.3$ & \\
\hline & & 402660101 & & 25 & 157409 & - & $52.9 \pm 14.5$ & $60.1 \pm 12.9$ & $-12.8 \pm 10.5$ & \\
\hline 37 & NGC 4157 & 203170101 & $\mathrm{H}$ & 30 & 2583 & TR & $5.7 \pm 11.3$ & $8.7 \pm 10.4$ & $85.2 \pm 11.3$ & \\
\hline 38 & NGC 4168 & 112550501 & S1.9 & 15 & 1053 & TR & $2.4 \pm 12.5$ & $75.8 \pm 12.3$ & $20.3 \pm 13.2$ & True-S2 (5) \\
\hline 39 & NGC 4214 & 035940201 & $\mathrm{H}$ & 9 & 1290 & TR & $-0.1 \pm 11.7$ & $14.6 \pm 11.9$ & $84.7 \pm 12.4$ & \\
\hline 40 & NGC 4235 & 204650201 & $\mathrm{~S} 1.2$ & 8 & 8137 & TR & $91.0 \pm 9.1$ & $1.1 \pm 7.6$ & $7.8 \pm 9.2$ & \\
\hline 41 & NGC 4254 & 147610101 & $\mathrm{H}$ & 11 & 1901 & - & $12.3 \pm 15.7$ & $10.5 \pm 14.8$ & $74.5 \pm 16.1$ & AGN (10) \\
\hline \multirow[t]{4}{*}{42} & NGC 4258 & 400560301 & S1.9 & 43 & 35016 & - & $34.6 \pm 15.2$ & $58.8 \pm 14.6$ & $6.4 \pm 11.1$ & \\
\hline & & 059140901 & & 9 & 7884 & - & $13.8 \pm 15.5$ & $73.5 \pm 15.6$ & $11.9 \pm 12.4$ & \\
\hline & & 059140101 & & 7 & 7752 & - & $10.5 \pm 14.1$ & $78.7 \pm 14.4$ & $10.7 \pm 12.1$ & \\
\hline & & 110920101 & & 11 & 14231 & TR & $4.3 \pm 7.9$ & $94.9 \pm 7.6$ & $1.1 \pm 5.5$ & \\
\hline \multirow[t]{2}{*}{43} & NGC 4261 & 056340101 & $\mathrm{~L} 2$ & 21 & 10740 & - & $-3.7 \pm 16.8$ & $14.6 \pm 13.6$ & $86.9 \pm 15.8$ & AGN (1) \\
\hline & & 502120101 & & 63 & 32201 & - & $-4.0 \pm 16.6$ & $15.2 \pm 12.0$ & $86.3 \pm 15.1$ & \\
\hline 44 & NGC 4278 & 205010101 & L1.9 & 20 & 34532 & - & $51.5 \pm 14.0$ & $-1.1 \pm 9.2$ & $50.6 \pm 14.2$ & $\mathrm{AGN}(1)$ \\
\hline 45 & NGC 4303 & 205360101 & $\mathrm{H}$ & 15 & 2624 & - & $10.6 \pm 15.7$ & $23.3 \pm 15.0$ & $64.0 \pm 16.6$ & AGN (6) \\
\hline 46 & NGC 4314 & 201690301 & $\mathrm{~L} 2$ & 14 & 1275 & - & $5.0 \pm 16.8$ & $29.8 \pm 16.4$ & $62.0 \pm 17.7$ & Non-AGN (1) \\
\hline 47 & NGC 4321 & 106860201 & $\mathrm{~T} 2$ & 9 & 2663 & - & $8.7 \pm 16.1$ & $24.4 \pm 15.0$ & $66.5 \pm 16.1$ & AGN (10) \\
\hline 48 & NGC 4374 & 673310101 & $\mathrm{~L} 2$ & 24 & 19790 & - & $-14.4 \pm 14.7$ & $4.1 \pm 12.8$ & $103.0 \pm 13.4$ & CT (11) \\
\hline 49 & NGC 4378 & 301650801 & S2 & 9 & 605 & - & $-6.7 \pm 17.7$ & $58.8 \pm 17.2$ & $42.4 \pm 17.7$ & True-S2 (5) \\
\hline 50 & NGC 4388 & 110930701 & S1.9 & 6 & 9801 & - & $10.8 \pm 12.2$ & $89.7 \pm 12.6$ & $-2.5 \pm 10.6$ & \\
\hline & & 675140101 & & 21 & 57292 & TR & $0.9 \pm 4.7$ & $99.1 \pm 4.5$ & $-0.0 \pm 3.3$ & \\
\hline 51 & NGC 4395 & 112522701 & $\mathrm{~S} 1.8$ & 5 & 6908 & - & $54.7 \pm 16.1$ & $28.6 \pm 14.3$ & $17.2 \pm 13.2$ & \\
\hline & & 142830101 & & 86 & 100135 & - & $90.1 \pm 11.5$ & $11.8 \pm 11.1$ & $-3.2 \pm 10.7$ & \\
\hline & & 112521901 & & 8 & 5453 & TR & $2.7 \pm 8.8$ & $95.8 \pm 8.7$ & $1.2 \pm 6.3$ & \\
\hline 52 & NGC 4414 & 402830101 & T2: & 16 & 3047 & - & $21.1 \pm 15.3$ & $19.5 \pm 15.6$ & $58.9 \pm 17.5$ & Non-AGN * \\
\hline 53 & NGC 4459 & 550540101 & $\mathrm{~T} 2:$ & 61 & 6839 & - & $27.0 \pm 16.4$ & $19.4 \pm 14.0$ & $55.2 \pm 17.5$ & Non-AGN (1) \\
\hline & & 550540201 & & 15 & 1543 & - & $3.2 \pm 15.6$ & $27.7 \pm 16.2$ & $67.8 \pm 17.1$ & \\
\hline 54 & NGC 4472 & 200130101 & $\mathrm{~S} 2::$ & 72 & 115802 & - & $-17.3 \pm 17.9$ & $2.1 \pm 16.5$ & $104.9 \pm 14.6$ & True-S2 (5) \\
\hline 55 & NGC 4486 & 114120101 & L2 & 24 & 361682 & - & $17.5 \pm 17.7$ & $-7.0 \pm 14.2$ & $79.5 \pm 16.1$ & AGN (1) \\
\hline 56 & NGC 4490 & 112280201 & $\mathrm{H}$ & 11 & 2399 & - & $15.2 \pm 16.1$ & $14.4 \pm 13.8$ & $70.6 \pm 15.2$ & \\
\hline & & 556300101 & & 18 & 5260 & TR & $4.5 \pm 9.5$ & $-0.7 \pm 7.5$ & $95.6 \pm 8.8$ & \\
\hline 57 & NGC 4494 & 071340301 & L2:: & 23 & 2101 & - & $24.4 \pm 16.8$ & $21.6 \pm 14.8$ & $53.1 \pm 16.6$ & AGN (1) \\
\hline 58 & NGC 4526 & 205010201 & $\mathrm{H}$ & 18 & 2739 & - & $21.4 \pm 16.4$ & $23.0 \pm 15.1$ & $57.3 \pm 16.6$ & AGN (12) \\
\hline 59 & NGC 4552 & 141570101 & T2: & 17 & 12193 & - & $-6.0 \pm 13.8$ & $-1.1 \pm 11.3$ & $99.7 \pm 13.1$ & $\operatorname{AGN}(1,10)$ \\
\hline 60 & NGC 4559 & 152170501 & $\mathrm{H}$ & 33 & 9950 & TR & $7.7 \pm 8.9$ & $0.0 \pm 6.4$ & $92.2 \pm 8.9$ & \\
\hline 61 & NGC 4565 & 112550301 & S1.9 & 8 & 1236 & TR & $-0.9 \pm 12.0$ & $79.4 \pm 12.0$ & $20.5 \pm 12.9$ & True-S2 (5) \\
\hline 62 & NGC 4569 & 200650101 & $\mathrm{~T} 2$ & 41 & 6720 & - & $19.5 \pm 14.5$ & $5.0 \pm 12.9$ & $74.9 \pm 14.6$ & AGN (10) \\
\hline 63 & NGC 4579 & 112840101 & S1.9 & 14 & 37493 & - & $63.1 \pm 13.0$ & $-3.2 \pm 9.1$ & $40.7 \pm 12.7$ & $\operatorname{AGN}(1,10)$ \\
\hline 64 & NGC 4594 & 084030101 & L2 & 15 & 11642 & - & $32.3 \pm 15.0$ & $1.6 \pm 11.1$ & $65.8 \pm 14.1$ & $\operatorname{AGN}(1,10)$ \\
\hline 65 & NGC 4636 & 111190701 & L1.9 & 50 & 91742 & - & $-12.7 \pm 16.4$ & $7.1 \pm 15.9$ & $93.4 \pm 16.3$ & Non-AGN (1) \\
\hline & & 111190201 & & 5 & 9876 & - & $-15.1 \pm 15.3$ & $10.7 \pm 14.6$ & $93.7 \pm 16.0$ & \\
\hline 66 & NGC 4639 & 112551001 & S1.0 & 8 & 2826 & TR & $84.0 \pm 11.4$ & $1.9 \pm 9.5$ & $13.9 \pm 11.3$ & \\
\hline
\end{tabular}


Table 1. continued.

\begin{tabular}{|c|c|c|c|c|c|c|c|c|c|c|}
\hline \multirow[b]{2}{*}{$\begin{array}{r}\text { Num } \\
(1)\end{array}$} & \multirow[b]{2}{*}{$\begin{array}{r}\text { Name } \\
(2)\end{array}$} & \multirow[b]{2}{*}{$\begin{array}{r}\text { ObsID } \\
\text { (3) }\end{array}$} & \multirow[b]{2}{*}{$\begin{array}{r}\text { Class } \\
(4)\end{array}$} & \multirow[b]{2}{*}{$\begin{array}{r}\text { Expos. } \\
(5)\end{array}$} & \multirow[b]{2}{*}{$\begin{array}{r}\text { Counts } \\
(6)\end{array}$} & \multicolumn{4}{|c|}{ ANN } & \multirow{2}{*}{$\begin{array}{r}\text { Comments } \\
\text { (11) }\end{array}$} \\
\hline & & & & & & $\begin{array}{r}\text { Train } \\
(7)\end{array}$ & $\begin{array}{l}v_{\mathrm{S} 1} \\
(8)\end{array}$ & $\begin{array}{r}v_{\mathrm{S} 1.8} \\
(9)\end{array}$ & $\begin{array}{r}v_{\mathrm{SB}} \\
(10)\end{array}$ & \\
\hline 67 & NGC 4698 & 651360401 & S2 & 27 & 1722 & - & $3.5 \pm 18.3$ & $47.3 \pm 17.8$ & $48.3 \pm 18.1$ & CT (11) \\
\hline 68 & NGC 4725 & 112550401 & S2: & 11 & 1333 & - & $13.4 \pm 17.4$ & $32.1 \pm 16.2$ & $49.9 \pm 17.7$ & True-S2 (5) \\
\hline \multirow{2}{*}{69} & NGC 4736 & 404980101 & L2 & 33 & 42014 & - & $30.3 \pm 13.5$ & $0.2 \pm 10.3$ & $67.2 \pm 14.5$ & $\operatorname{AGN}(1,10)$ \\
\hline & & 094360601 & & 8 & 11071 & - & $26.1 \pm 13.6$ & $3.7 \pm 10.8$ & $71.4 \pm 14.2$ & \\
\hline 70 & NGC 4845 & 658400601 & $\mathrm{H}$ & 14 & 83763 & - & $2.9 \pm 6.1$ & $96.7 \pm 6.0$ & $-0.0 \pm 3.2$ & AGN (13) \\
\hline 71 & NGC 5005 & 110930501 & L1.9 & 8 & 2952 & - & $2.8 \pm 16.1$ & $13.4 \pm 13.5$ & $82.7 \pm 16.2$ & CT $(4,11)$ \\
\hline 72 & NGC 5033 & 094360501 & S1.5 & 8 & 21897 & TR & $93.9 \pm 7.5$ & $-0.1 \pm 5.4$ & $6.2 \pm 7.6$ & $\operatorname{AGN}(1,10)$ \\
\hline \multirow[t]{2}{*}{73} & NGC 5055 & 405080301 & $\mathrm{~T} 2$ & 5 & 1675 & - & $9.5 \pm 17.9$ & $21.8 \pm 16.3$ & $65.4 \pm 17.0$ & AGN (10) \\
\hline & & 405080501 & & 2 & 812 & _- & $-3.7 \pm 17.2$ & $44.2 \pm 17.0$ & $51.3 \pm 18.8$ & \\
\hline 74 & NGC 5194 & 112840201 & $\mathrm{~S} 2$ & 17 & 11622 & - & $20.8 \pm 14.3$ & $8.4 \pm 13.2$ & $65.0 \pm 15.3$ & CT $(2,4)$ \\
\hline \multirow[t]{2}{*}{75} & NGC 5195 & 303420201 & L2: & 20 & 3318 & - & $-13.9 \pm 17.1$ & $20.2 \pm 17.8$ & $91.5 \pm 19.3$ & AGN (10) \\
\hline & & 212480801 & & 22 & 3764 & - & $4.3 \pm 14.8$ & $12.5 \pm 15.1$ & $83.4 \pm 15.5$ & \\
\hline \multirow[t]{5}{*}{76} & NGC 5204 & 142770301 & $\mathrm{H}$ & 3 & 2771 & TR & $6.2 \pm 10.6$ & $2.4 \pm 9.1$ & $91.5 \pm 10.7$ & \\
\hline & & 150650301 & & 4 & 4484 & - & $21.8 \pm 13.4$ & $0.7 \pm 11.6$ & $76.3 \pm 14.3$ & \\
\hline & & 405690201 & & 25 & 27564 & - & $32.4 \pm 14.1$ & $-6.2 \pm 10.9$ & $73.0 \pm 14.2$ & \\
\hline & & 405690101 & & 7 & 9860 & - & $28.8 \pm 14.3$ & $-7.1 \pm 11.1$ & $77.4 \pm 14.0$ & \\
\hline & & 405690501 & & 19 & 16050 & - & $42.0 \pm 14.0$ & $-0.9 \pm 10.1$ & $59.9 \pm 14.2$ & \\
\hline 77 & NGC 5248 & 655380401 & $\mathrm{H}$ & 7 & 941 & TR & $-2.2 \pm 12.2$ & $14.3 \pm 12.3$ & $86.6 \pm 12.6$ & \\
\hline 78 & NGC 5273 & 112551701 & S1.5 & 9 & 15529 & TR & $96.8 \pm 7.6$ & $2.9 \pm 6.9$ & $0.4 \pm 7.1$ & \\
\hline 79 & NGC 5322 & 071340501 & $\mathrm{~L} 2::$ & 13 & 1344 & - & $7.7 \pm 17.5$ & $29.9 \pm 16.5$ & $57.2 \pm 17.5$ & AGN (14) \\
\hline 80 & NGC 5363 & 201670201 & L2 & 11 & 1850 & - & $17.1 \pm 17.3$ & $19.0 \pm 16.1$ & $62.0 \pm 16.4$ & AGN (1) \\
\hline \multirow[t]{3}{*}{81} & NGC 5548 & 109960101 & S1.5 & 15 & 309750 & - & $100.3 \pm 7.0$ & $0.7 \pm 5.9$ & $-2.2 \pm 7.5$ & \\
\hline & & 089960301 & & 47 & 1185380 & - & $99.7 \pm 6.2$ & $-0.3 \pm 5.1$ & $0.9 \pm 6.1$ & \\
\hline & & 089960401 & & 18 & 636996 & TR & $100.0 \pm 3.2$ & $0.0 \pm 2.4$ & $0.0 \pm 3.1$ & \\
\hline \multirow[t]{4}{*}{82} & NGC 5746 & 651890101 & $\mathrm{~T} 2$ & 42 & 2986 & - & $30.8 \pm 17.8$ & $29.5 \pm 16.6$ & $39.4 \pm 15.1$ & AGN (1) \\
\hline & & 651890201 & & 28 & 1814 & - & $19.9 \pm 18.7$ & $33.3 \pm 16.0$ & $44.0 \pm 19.1$ & \\
\hline & & 651890301 & & 66 & 4867 & - & $37.3 \pm 17.2$ & $22.2 \pm 15.5$ & $41.6 \pm 15.7$ & \\
\hline & & 651890401 & & 54 & 3692 & - & $36.4 \pm 17.2$ & $33.4 \pm 14.4$ & $32.6 \pm 15.4$ & \\
\hline \multirow[t]{3}{*}{83} & NGC 5813 & 302460101 & L2: & 19 & 25523 & - & $-20.5 \pm 15.8$ & $7.8 \pm 15.2$ & $100.2 \pm 15.6$ & Non-AGN (1) \\
\hline & & 554680201 & & 43 & 59102 & - & $-20.9 \pm 16.4$ & $5.9 \pm 14.7$ & $99.6 \pm 15.4$ & \\
\hline & & 554680301 & & 42 & 58293 & - & $-19.4 \pm 16.0$ & $6.6 \pm 14.9$ & $99.7 \pm 15.8$ & \\
\hline \multirow[t]{2}{*}{84} & NGC 5846 & 021540501 & $\mathrm{~T} 2:$ & 10 & 10036 & - & $-22.9 \pm 16.2$ & $6.6 \pm 14.3$ & $102.9 \pm 15.2$ & Non-AGN (1) \\
\hline & & 021540101 & & 25 & 25881 & - & $-20.6 \pm 15.9$ & $5.3 \pm 14.1$ & $102.1 \pm 14.5$ & \\
\hline 85 & NGC 5982 & 673770401 & $\mathrm{~L} 2::$ & 8 & 1920 & - & $-12.8 \pm 17.1$ & $16.8 \pm 16.1$ & $91.0 \pm 18.3$ & Non-AGN (14) \\
\hline \multirow[t]{2}{*}{86} & NGC 6217 & 400920101 & $\mathrm{H}$ & 6 & 1011 & - & $-16.5 \pm 16.0$ & $43.3 \pm 16.4$ & $66.0 \pm 18.5$ & AGN (15) \\
\hline & & 400920201 & & 7 & 1137 & - & $-12.2 \pm 16.9$ & $23.5 \pm 16.3$ & $81.8 \pm 16.8$ & \\
\hline \multirow[t]{2}{*}{87} & NGC 6482 & 304160801 & $\mathrm{~T} 2 / \mathrm{S}$ & 4 & 3163 & - & $-28.6 \pm 17.8$ & $6.4 \pm 15.5$ & $110.7 \pm 16.7$ & Non-AGN (1) \\
\hline & & 304160401 & & 6 & 4811 & - & $-25.1 \pm 17.5$ & $7.5 \pm 14.8$ & $107.4 \pm 15.4$ & \\
\hline 88 & NGC 6703 & 601830401 & $\mathrm{~L} 2::$ & 14 & 1154 & - & $4.9 \pm 18.9$ & $43.9 \pm 17.5$ & $49.8 \pm 17.6$ & Non-AGN ^ \\
\hline \multirow[t]{4}{*}{89} & NGC 6946 & 200670301 & $\mathrm{H}$ & 7 & 1108 & - & $-1.2 \pm 16.2$ & $21.0 \pm 16.5$ & $76.5 \pm 15.7$ & AGN (16) \\
\hline & & 200670401 & & 4 & 703 & - & $-4.6 \pm 17.1$ & $38.0 \pm 16.8$ & $61.4 \pm 17.9$ & \\
\hline & & 500730101 & & 19 & 2281 & - & $-0.8 \pm 16.5$ & $17.0 \pm 14.8$ & $82.5 \pm 16.1$ & \\
\hline & & 500730201 & & 26 & 4205 & _- & $9.5 \pm 15.5$ & $9.2 \pm 13.0$ & $83.2 \pm 16.1$ & \\
\hline 90 & NGC 7626 & 149240101 & L2:: & 33 & 3041 & _- & $-14.4 \pm 17.7$ & $27.5 \pm 18.1$ & $82.6 \pm 17.3$ & AGN (17) \\
\hline
\end{tabular}




\section{Appendix A: Hydrogen column densities}

We have estimated the hydrogen column densities, $N_{\mathrm{H}}$, for the observations in our sample with $v_{\mathrm{SB}}<10$ with the purpose of comparing them with $v_{\mathrm{S} 1.8}$ (see Fig. 6 and Sect. 6.1). The spectral fitting was performed using XSPEC v12.7.1. The spectra were binned to a minimum of 20 counts per spectral bin before background subtraction to use the $\chi^{2}$ statistics. The task GRPPHA included in the FTOOLS software was used for this purpose. We used the simplest fit that represents the data, a single power-law. Therefore, we fitted the spectra in the $2-10 \mathrm{keV}$ energy band to a single power-law, with a fixed spectral index of $\Gamma=2.1$. This power-law is attenuated by an absorber, ZWABS within Xspec, and three Gaussian profiles centred on 6.4, 6.7, and 6.95 keV were added to include the plausible existence of the neutral and ionised iron lines. Note that the width of the lines was fixed to the spectral resolution of $X M M$-Newton for the ionised lines but was let free to vary for the neutral FeK $\alpha$ line. The resulting $N_{\mathrm{H}}$ estimates are reported in Table A.1. This table also includes the $N_{\mathrm{H}}$ values reported in the literature for the same observations. We only included the spectral fittings corresponding to the same observations included in our analysis because many of these objects are highly variable. Our $N_{\mathrm{H}}$ values agree with those from literature for high $N_{\mathrm{H}}$. Discrepancies up to a factor of $\sim 3$ are found for lower $N_{\mathrm{H}}$ values. The spectral fittings performed in the literature have the advantage of providing a more realistic modelling of the spectra. However, we have found very few of them, and the modelling performed is not the same in all the cases. Our simple spectral fitting has the advantage of being homogenous and it is available for all the objects with $v_{\mathrm{SB}}<10$. Thus, our simple spectral fitting is better suited for the comparison of $N_{\mathrm{H}}$ and $v_{\mathrm{S} 1.8}$ (see Fig. 6).

Table A.1. Logarithmic of the hydrogen column densities, $\log \left(N_{\mathrm{H}}\right)$, for observations in our sample with $v_{\mathrm{SB}}<10$.

\begin{tabular}{|c|c|c|c|c|}
\hline \multirow[t]{2}{*}{ Name } & \multirow[t]{2}{*}{ ObsID } & \multicolumn{3}{|c|}{$\log \left(N_{\mathrm{H}}\right)$} \\
\hline & & Own & Literature & Ref. \\
\hline \multirow[t]{4}{*}{ NGC 1052} & 553300401 & 23.0 & 23.1 & (B) \\
\hline & 093630101 & 23.0 & & \\
\hline & 553300301 & 23.0 & & \\
\hline & 306230101 & 23.0 & & \\
\hline NGC 2273 & 140951001 & 23.2 & 24.0 & (B) \\
\hline \multirow[t]{2}{*}{ NGC 3227} & 101040301 & 22.9 & 22.8 & (A) \\
\hline & 400270101 & 22.2 & 22.0 & (T) \\
\hline \multirow[t]{6}{*}{ NGC 3516} & 107460601 & 22.5 & & \\
\hline & 107460701 & 22.6 & 22.0 & (A) \\
\hline & 401210501 & 22.3 & 22.6 & (T) \\
\hline & 401210601 & 22.4 & 22.7 & (T) \\
\hline & 401210401 & 22.2 & 22.6 & (T) \\
\hline & 401211001 & 22.2 & 22.5 & (T) \\
\hline \multirow{15}{*}{ NGC 4051} & 157560101 & 22.4 & 22.8 & (T) \\
\hline & 606321601 & 22.0 & & \\
\hline & 606320201 & 22.1 & & \\
\hline & 606321901 & 22.6 & & \\
\hline & 606322001 & 22.4 & & \\
\hline & 606320301 & 22.1 & & \\
\hline & 606320401 & 22.6 & & \\
\hline & 606321501 & 22.2 & & \\
\hline & 606321701 & 22.3 & & \\
\hline & 606322301 & 22.4 & & \\
\hline & 606322201 & 22.5 & & \\
\hline & 606321401 & 22.2 & & \\
\hline & 606322101 & 22.7 & & \\
\hline & 606321801 & 22.5 & & \\
\hline & 606320101 & 22.3 & & \\
\hline NGC 4138 & 112551201 & 23.0 & 22.9 & (A) \\
\hline \multirow{8}{*}{ NGC 4151} & 112310101 & 22.9 & 22.8 & (B) \\
\hline & 112830501 & 22.9 & 22.9 & (T) \\
\hline & 112830201 & 22.9 & 22.9 & (T) \\
\hline & 143500301 & 22.8 & 22.8 & (T) \\
\hline & 402660201 & 23.0 & 23.0 & (T) \\
\hline & 143500201 & 22.9 & 22.9 & (T) \\
\hline & 143500101 & 22.9 & & \\
\hline & 402660101 & 23.1 & & \\
\hline NGC 4235 & 204650201 & 22.3 & & \\
\hline \multirow{2}{*}{ NGC 4258} & 400560301 & 22.9 & 22.8 & (A) \\
\hline & 110920101 & 23.0 & 22.9 & (C) \\
\hline \multirow{2}{*}{ NGC 4388} & 110930701 & 23.5 & 23.6 & (B) \\
\hline & 675140101 & 23.4 & & \\
\hline \multirow{2}{*}{ NGC 4395} & 142830101 & 22.6 & 22.0 & (A) \\
\hline & 112521901 & 23.0 & 22.7 & (T) \\
\hline NGC 5033 & 094360501 & 22.1 & 23.5 & (C) \\
\hline NGC 5273 & 112551701 & 22.4 & 21.9 & (C) \\
\hline \multirow[t]{3}{*}{ NGC 5548} & 109960101 & 22.2 & & \\
\hline & 089960301 & 22.1 & & \\
\hline & 089960401 & 22.1 & & \\
\hline
\end{tabular}

References. (A) Akylas \& Georgantopoulos (2009), (B) Brightman \& Nandra (2011), (C) Cappi et al. (2006), and (T) Tombesi et al. (2010). 
Appendix B: Catalogue of spectra
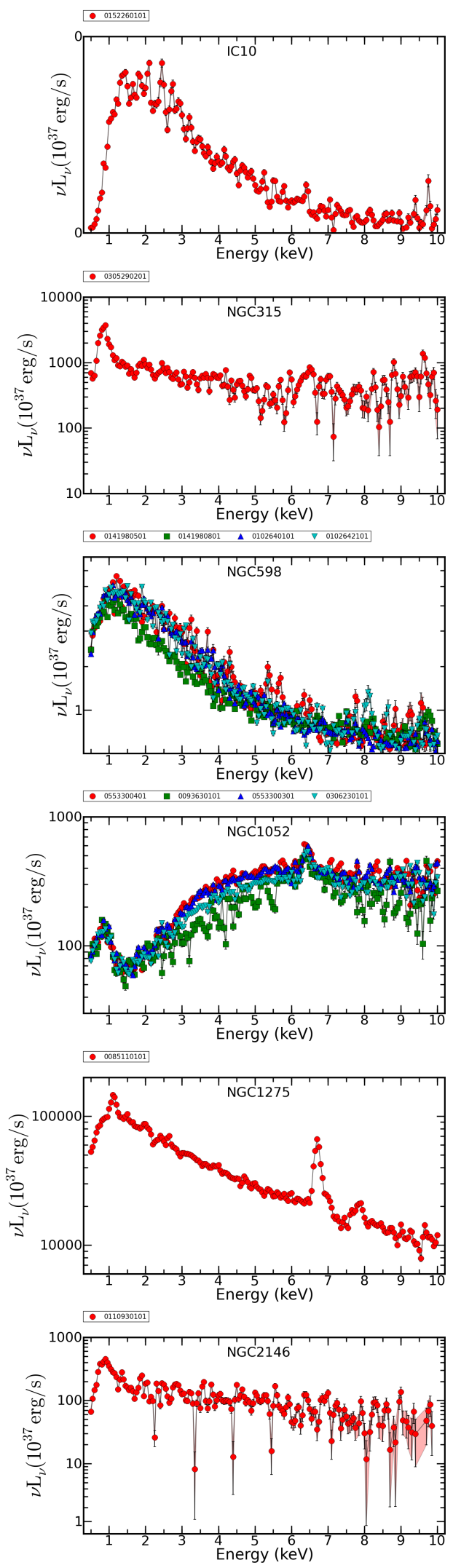
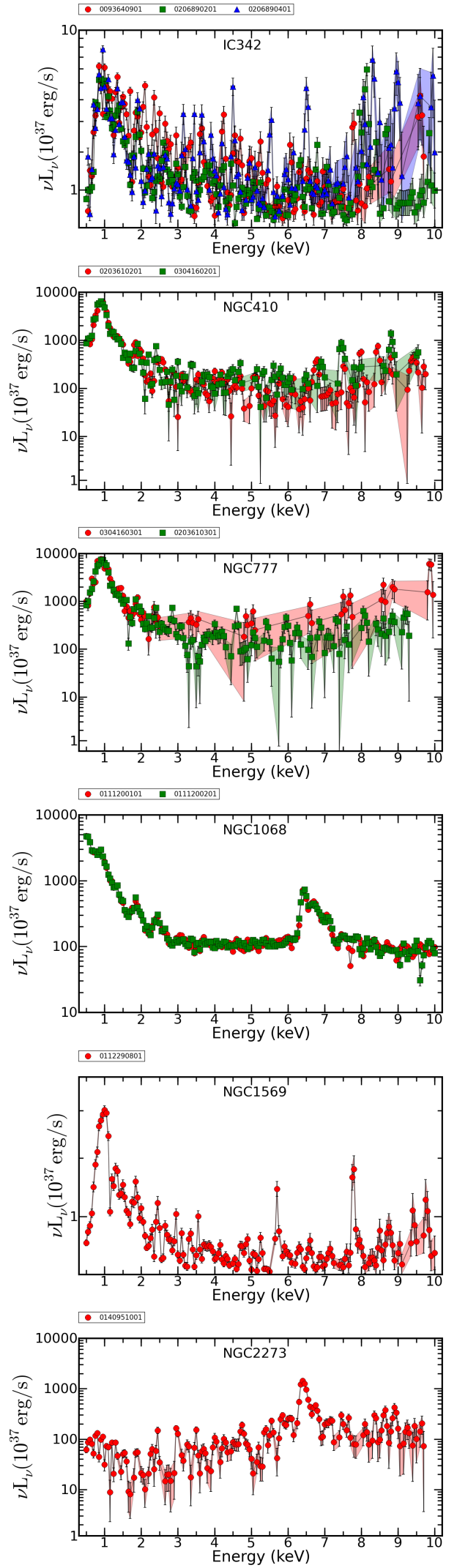

Fig. B.1. Flux-calibrated spectra of the sample. The grey-shaded region shows the error bars of the spectra. All the observations of the same objects are shown in the same panel using different colours. 
O. González-Martín et al.: Synapses of active galactic nuclei
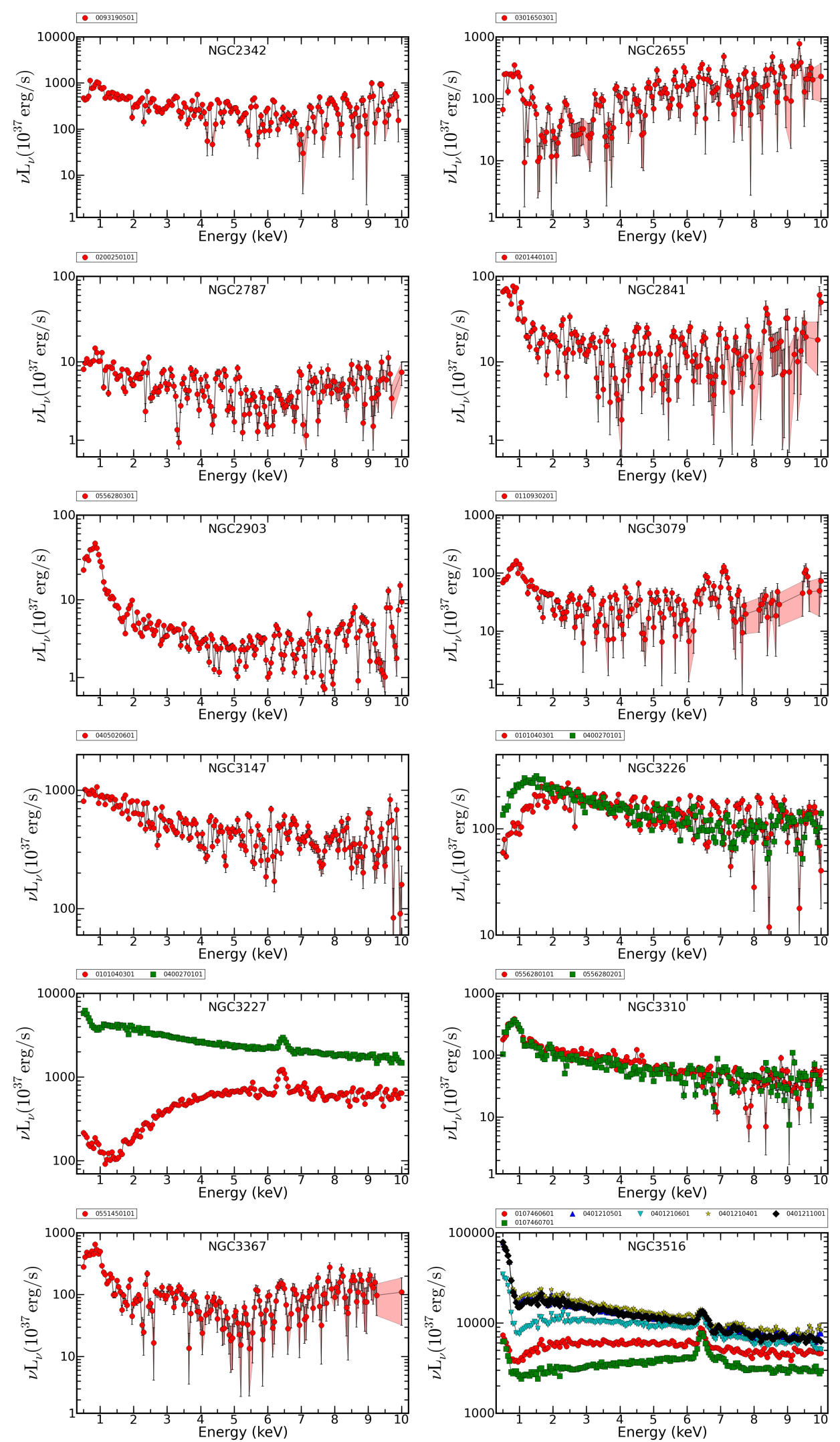

Fig. B.1. Flux-calibrated spectra of the sample (continued). 
A\&A 567, A92 (2014)
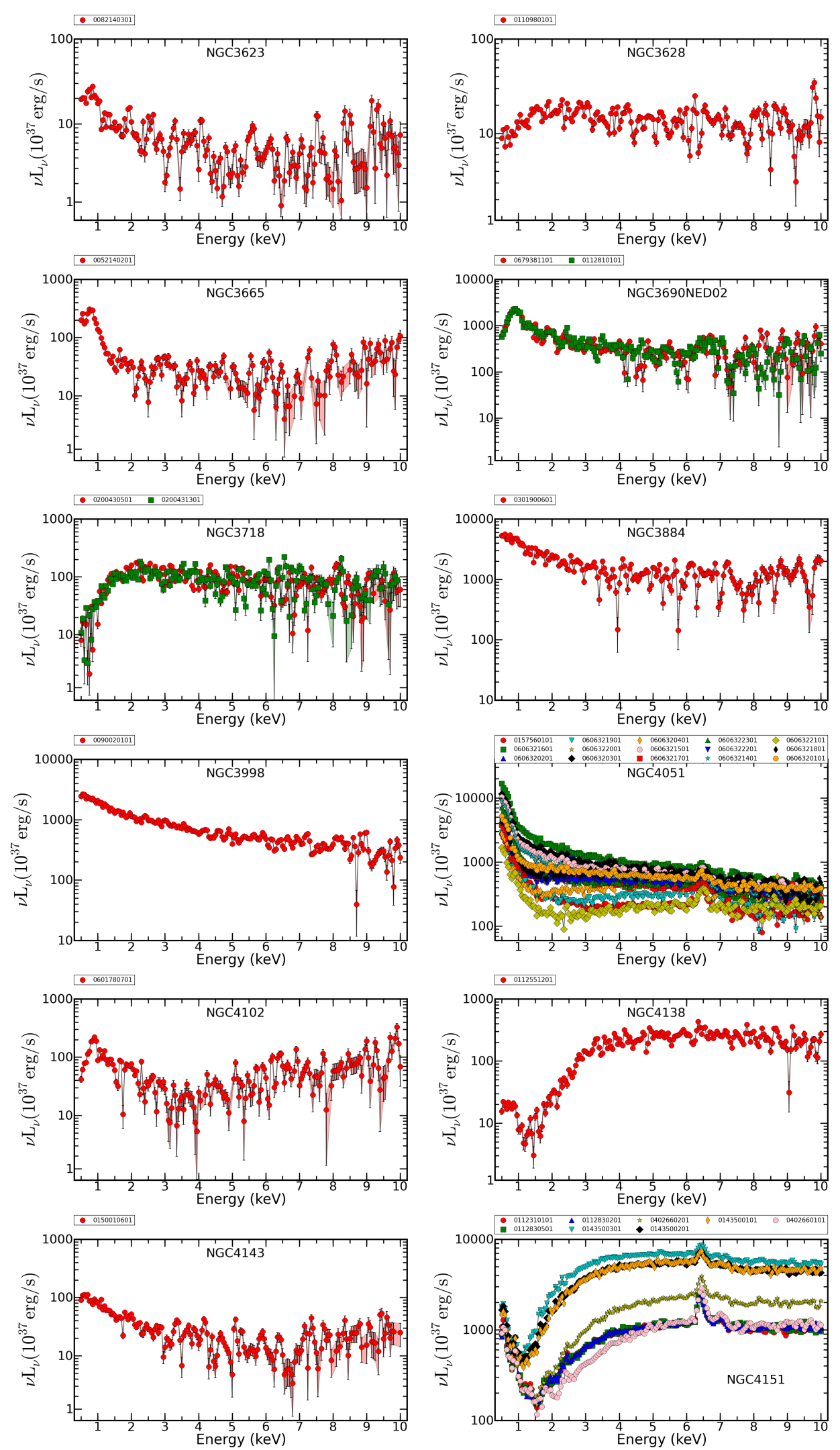

Fig. B.1. Flux-calibrated spectra of the sample (continued). 
O. González-Martín et al.: Synapses of active galactic nuclei
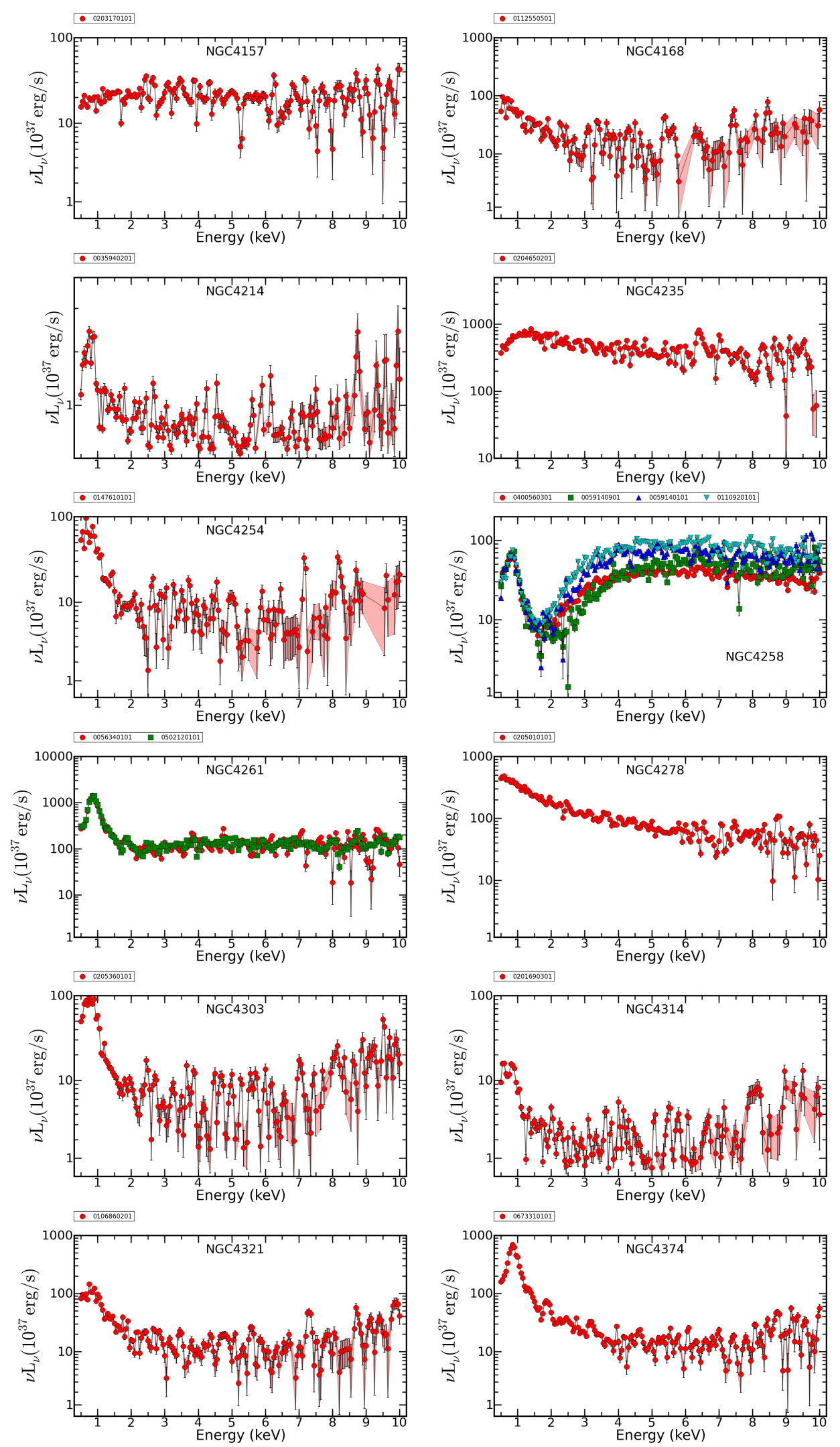

Fig. B.1. Flux-calibrated spectra of the sample (continued). 

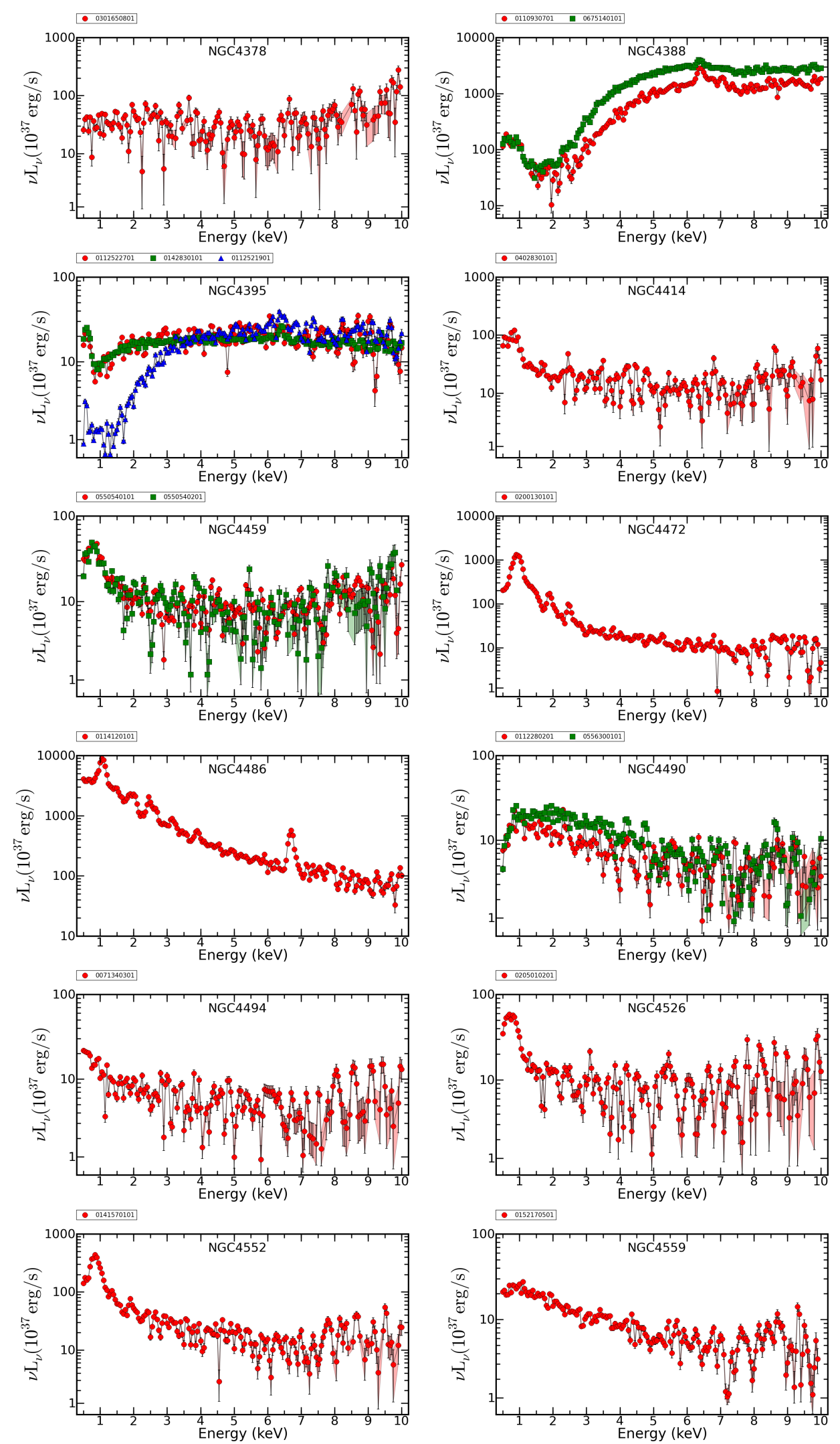

Fig. B.1. Flux-calibrated spectra of the sample (continued). 
O. González-Martín et al.: Synapses of active galactic nuclei
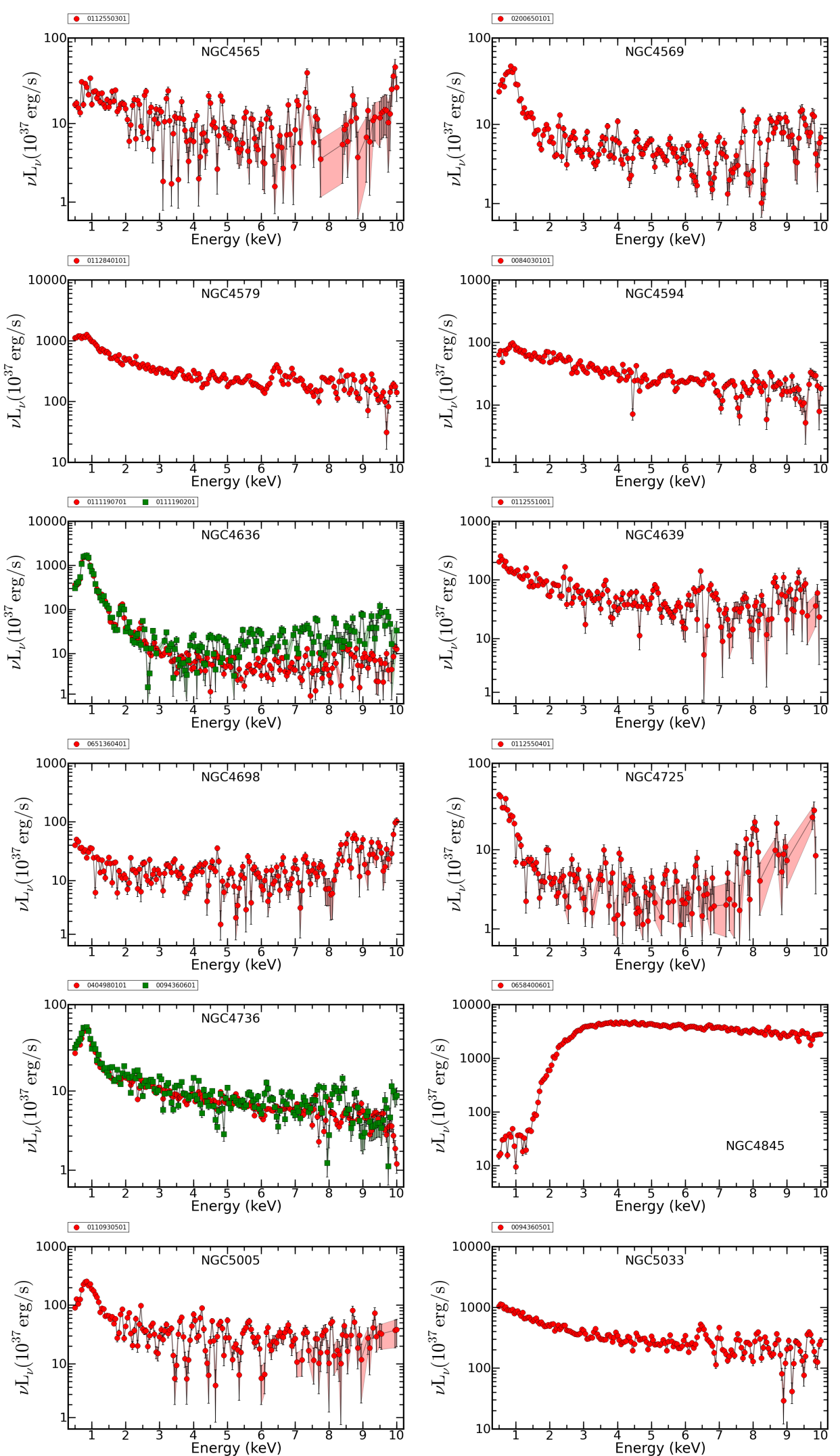

Fig. B.1. Flux-calibrated spectra of the sample (continued). 

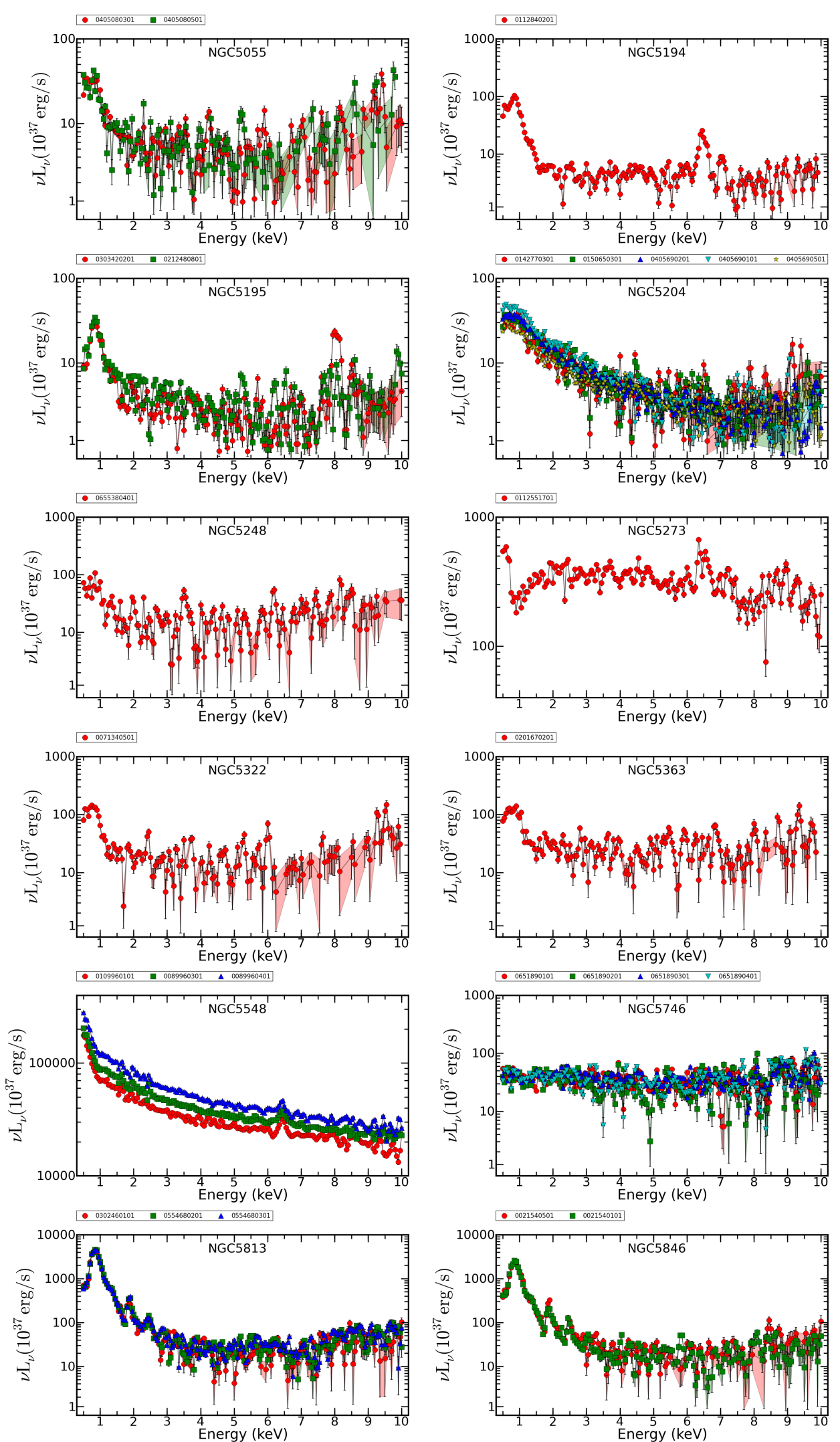

Fig. B.1. Flux-calibrated spectra of the sample (continued). 
O. González-Martín et al.: Synapses of active galactic nuclei
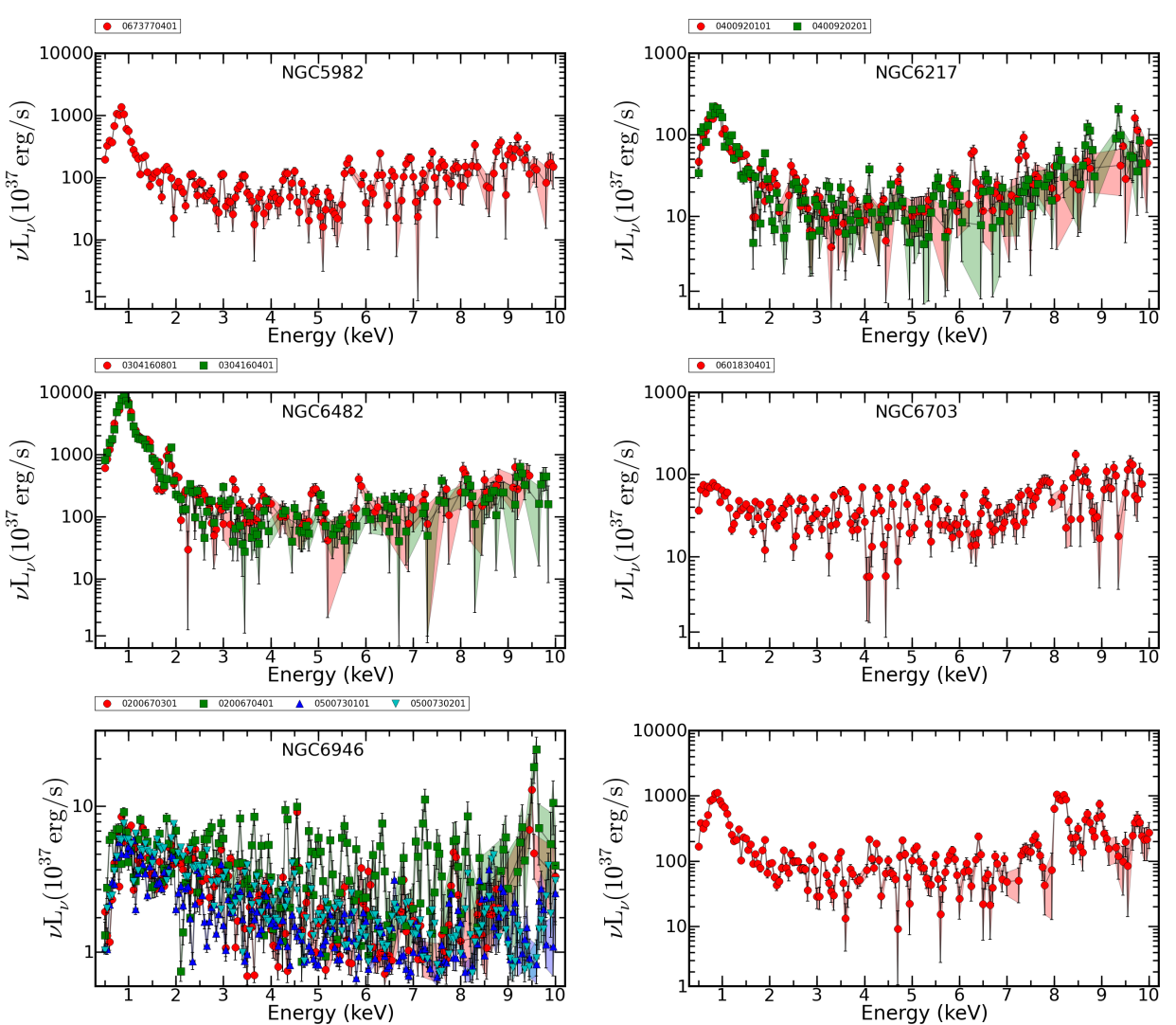

Fig. B.1. Flux-calibrated spectra of the sample (continued). 